\title{
Synthesis of Chiral 1,3-Dienes through Ring-Closing Metathesis of Enantioenriched Enynes: Potential Precursors of Morphane Analogs
}

\author{
MARÍA JESÚS GARCIA-MUÑOZ, ${ }^{1,2,3}$ ANA SIRVENT, ${ }^{1,2,3}$ FRANCISCO FOUBELO ${ }^{1,2,3}$ and MIGUEL YUS ${ }^{1,3}$ \\ ${ }^{1}$ Departamento de Química Orgánica, Facultad de Ciencias, Universidad de Alicante, Apdo. 99, 03080 Alicante, Spain \\ ${ }^{2}$ Instituto de Síntesis Orgánica/ISO,Universidad de Alicante, Apdo. 99, 03080 Alicante, Spain \\ ${ }^{3}$ Centro de Innovación en Química Avanzada (ORFEO-CINQA), Universidad de Alicante, Apdo. 99, 03080 Alicante, Spain
}

Manuscript received on September 27, 2017; accepted for publication on October 24, 2017

\begin{abstract}
A simple methodology for the synthesis of enynes by indium mediated diastereoselective allylation of aromatic $\mathrm{N}$-tert-butanesulfinylimines bearing alkenyl groups at ortho-position with allyl bromide has been developed. The addition of the allyl indium intermediate to the chiral imine took place with excellent diastereoselectivity. Ruthenium-catalyzed ring-closing metathesis of the resulting enynes provided the expected cyclic 1,3-dienes in good to moderate yields. These chiral dienes are potential precursors of biologically and pharmacologically active morphane derivatives.
\end{abstract}

Key words: allylation, imines, dienes, enynes, metathesis, alkaloids.

\section{INTRODUCTION}

Ring closing metathesis (Schmalz 1995) is an important tool in organic synthesis because cyclic unsaturated compounds could be accessed from linear hydrocarbons with double or triple bonds at the terminal positions. When the ring closing metathesis is performed on an enyne system, the resulting cyclic 1,3-dienes with one exocyclic double bond are of special interest (Kaliappan 2005). These dienes are versatile synthetic intermediates and could be involved in a wide

Correspondence to: Francisco Foubelo

E-mail: foubelo@ua.es

Miguel Yus

E-mail: yus@ua.es

*Contribution on the centenary of the Brazilian Academy of Sciences. variety of process, such as cycloaddition reactions, electrophilic additions, etc. The first ring closing metathesis of enynes was performed by Katz and Sivavec in 1985 by means of a tungsten Fischer carbene complex (Katz and Sivavec 1985). More recently, Grubbs developed more efficient catalysts of ruthenium to perform olefin metathesis in different types of solvents (Vougioukalakis and Grubbs 2010). In addition, these catalysts were air-tolerant, avoiding the tedious work under inert atmospheres, and also compatible with a wide range of functional groups (Hoveyda et al. 2004). In 2010, Tan and co-workers studied the ring-closing metathesis of chiral propargyl amines bearing allyl and tert-butanesulfinyl groups bonded to the nitrogen atom, using a second generation ruthenium catalyst (Bauer et al. 2010). In this way, vinylpyrrolines were obtained as reaction products 
in high yields (Figure 1a). The group of Fustero reported the synthesis of enantiopure cyclic dienes through the ring-closing metathesis of homoallylic benzylic alcohols with an alkenyl group at orthoposition of the aromatic ring (Rodriguez et al. 2016), using Grubbs second generation catalyst in the presence of 1,7-octadiene as additive (Figure 1b). More recently, the same group performed the ring-closing metathesis of structural related $\mathrm{N}$-tertbutanesulfinyl amino derivatives under similar reaction conditions (Lazaro et al. 2017), producing the expected dienes in high yields (Figure 1c). On the other hand, our research group reported the stereoselective allylation (Foubelo and Yus 2004) and propargylation (Garcia-Muñoz et al. 2013) of $N$-tert-butanesulfinyl aldimines and ketimines (Sirvent et al. 2012) with brominated precursors by means of indium metal, leading to homoallylic and homopropargylic amine derivatives, respectively, with high diastereoselectivity. These compounds were used as precursors in the synthesis of natural products (Foubelo and Yus 2014) and other structurally diverse nitrogen-containing compounds (Garcia-Muñoz et al. 2016). Continuing our interest in this topic, and prompted by the latest results from the group of Fustero, we report herein our approach to the synthesis enantioenriched 1,3-dienes with a $N$-tert-butanesulfinamide group through an enyne ring-closing metathesis.

\section{EXPERIMENTAL}

\section{GENERAL EXPERIMENTAL PROCEDURES}

$\left(R_{\mathrm{S}}\right)$-tert-Butanesulfinamide was a gift of Medalchemy ( $>99 \%$ ee by chiral HPLC on a Chiracel AS column, 90:10 $n$-hexane $/ i$-PrOH, 1.2 $\mathrm{mL} / \mathrm{min}, 1=222 \mathrm{~nm}$ ). TLC was performed on silica gel $60 \mathrm{~F}_{254}$, using aluminium plates and visualized with phosphomolybdic acid (PMA) stain. Flash chromatography was carried out on handpacked columns of silica gel 60 (230- 400 mesh). Melting points are uncorrected. Optical rotations were

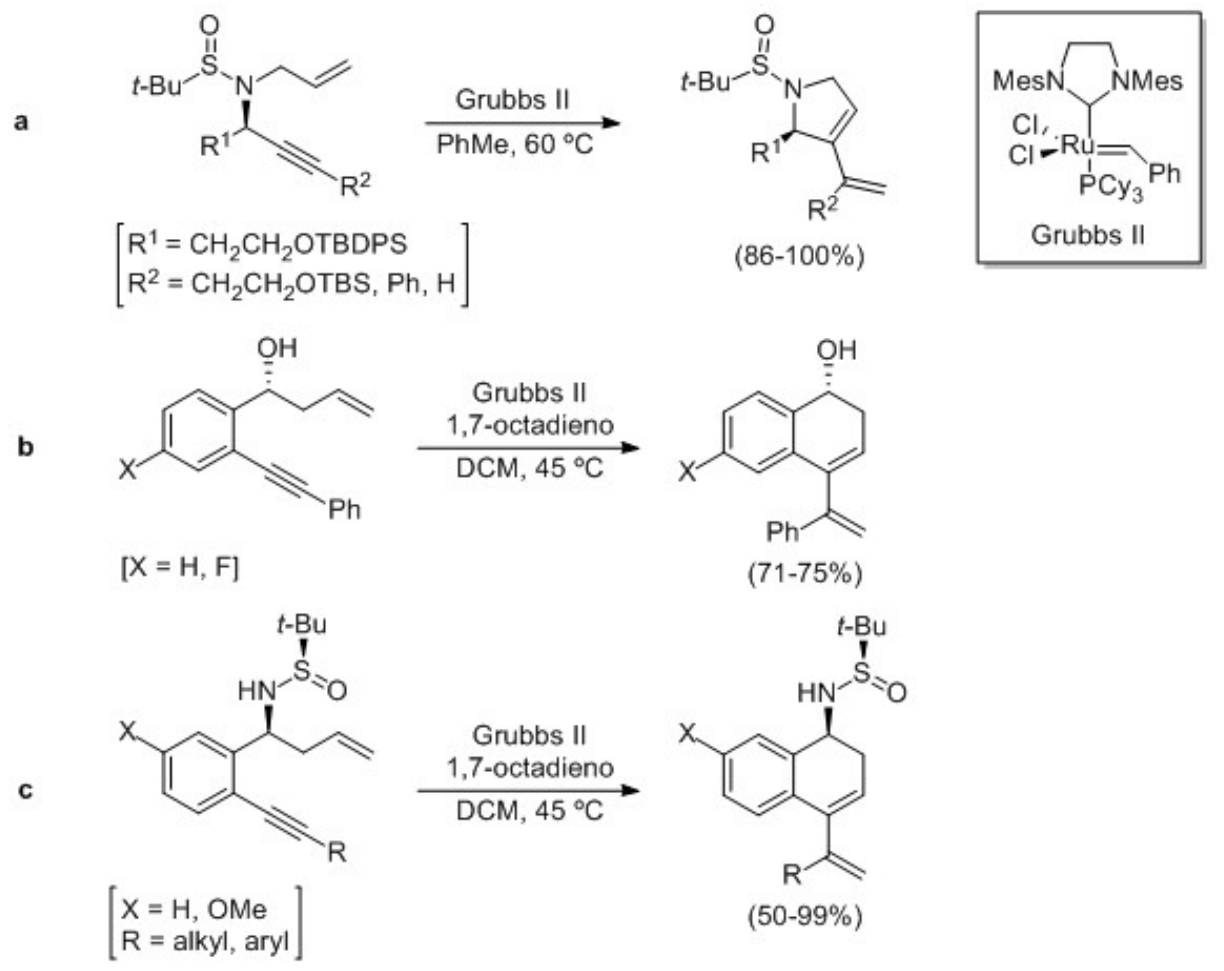

Figure 1 - Examples of ring-closing metathesis in $\mathrm{N}$-tert-butasulfinamides bearing enyne moiety. 
measured using a polarimeter with a thermally jacketed $5 \mathrm{~cm}$ cell at approximately $20{ }^{\circ} \mathrm{C}$ and concentrations (c) are given in $\mathrm{g} / 100 \mathrm{~mL}$. Infrared analyses were performed with a spectrophotometer equipped with an ATR component; wave numbers are given in $\mathrm{cm}^{-1}$. Low-resolution mass spectra (EI) were obtained at $70 \mathrm{eV}$, and fragment ions in $\mathrm{m} / \mathrm{z}$ with relative intensities (\%) in parentheses. Highresolution mass spectra (HRMS) were also carried out in the electron impact mode (EI) at $70 \mathrm{eV}$ and on an apparatus equipped with a time of flight (TOF) analyzer and the samples were ionized by ESI techniques and introduced through an ultra-high pressure liquid chromatography (UPLC) model. ${ }^{1} \mathrm{H}$ NMR spectra were recorded at 300 or $400 \mathrm{MHz}$ for ${ }^{1} \mathrm{H}$ NMR and 75 or $100 \mathrm{MHz}$ for ${ }^{13} \mathrm{C}$ NMR, using $\mathrm{CDCl}_{3}$ as the solvent and TMS as internal standard $(0.00 \mathrm{ppm})$. The data are being reported as: $\mathrm{s}=$ singlet, $\mathrm{d}=$ doublet, $\mathrm{t}=$ triplet, $\mathrm{q}=$ quadruplet, $\mathrm{m}=$ multiplet or unresolved, br $\mathrm{s}=$ broad signal, coupling constant(s) in $\mathrm{Hz}$, integration. ${ }^{13} \mathrm{C}$ NMR spectra were recorded with ${ }^{1} \mathrm{H}$-decoupling at 100 $\mathrm{MHz}$ and referenced to $\mathrm{CDCl}_{3}$ at $77.16 \mathrm{ppm}$. DEPT-135 experiments were performed to assign $\mathrm{CH}, \mathrm{CH}_{2}$ and $\mathrm{CH}_{3}$.

\section{SYNTHESIS OF 2-VINYLBENZALDEHYDE (3A)}

To a solution of $o$-bromostyrene (2a, $0.915 \mathrm{~g}, 0.626$ $\mathrm{mL}, 5.0 \mathrm{mmol})$ in anhydrous THF $(15 \mathrm{~mL})$ was added drop wise a $2.5 \mathrm{M}$ solution of $n$-BuLi in hexane $(2.2 \mathrm{~mL}, 5.5 \mathrm{mmol})$ at $-78^{\circ} \mathrm{C}$. The reaction mixture was stirred at this temperature for $1 \mathrm{~h}$, and after that, dry dimethylformamide (DMF, $0.456 \mathrm{~g}$, $0.483 \mathrm{~mL}, 6.25 \mathrm{mmol}$ ) was also added dropwise. Stirring was continued for $20 \mathrm{~min}$ at the same temperature and the reaction was hydrolyzed with a saturated aqueous $\mathrm{NH}_{4} \mathrm{Cl}$ solution $(6 \mathrm{~mL})$, and extracted with diethyl ether $(3 \times 10 \mathrm{~mL})$. The organic layer was washed with brine $(2 \times 10 \mathrm{~mL})$, dried over anhydrous magnesium sulfate and evaporated (15 Torr) to yield almost pure 2-vinylbenzaldehyde (3a) as a pale yellow oil $(0.587 \mathrm{~g}, 4.45 \mathrm{mmol}$, $89 \%$ ): $R_{\mathrm{F}} 0.50$ (hexane/AcOEt 10:1); IR (film) $v$ 2917, 2858, 2750, 1691, 1595, 1565, 1479, 1205, 1186, 922, 861, 770, $741 \mathrm{~cm}^{-1}$; ${ }^{1} \mathrm{H}-\mathrm{NMR}(400 \mathrm{MHz}$, $\left.\mathrm{CDCl}_{3}\right) \delta 10.28(1 \mathrm{H}, \mathrm{s}, \mathrm{COH}), 7.82(1 \mathrm{H}, \mathrm{dt}, J=$ 7.5, 1.0 Hz, ArH), 7.59-7.49 (3H, m, ArH), 7.45$7.38\left(1 \mathrm{H}, \mathrm{m}, \mathrm{CH}=\mathrm{CH}_{2}\right), 5.70(1 \mathrm{H}, \mathrm{dd}, J=17.4$, $1.2 \mathrm{~Hz}, \mathrm{CH}=\mathrm{CH} H), 5.51(1 \mathrm{H}, \mathrm{dd}, J=11.0,1.2 \mathrm{~Hz}$, $\mathrm{CH}=\mathrm{CHH}) ;{ }^{13} \mathrm{C}-\mathrm{NMR}\left(101 \mathrm{MHz}, \mathrm{CDCl}_{3}\right) \delta 192.3$ $(\mathrm{CH}), 140.4(\mathrm{C}), 133.7,133.3(\mathrm{CH}), 132.8(\mathrm{C})$, 131.1, 127.8, $127.4(\mathrm{CH}), 119.3\left(\mathrm{CH}_{2}\right)$; LRMS (EI) $\mathrm{m} / \mathrm{z} 132\left(\mathrm{M}^{+}, 31 \%\right), 131$ (14), 104 (21), 103 (24), 91 (17), 77 (15), 71 (24), 70 (21), 69 (18), 57 (28), 55 (20), 45 (19), 43 (100).

GENERAL PROCEDURE FOR THE PREPARATION OF ALDEHYDES 9

To a solution of the corresponding $o$-bromobenzaldehyde $3(7.0 \mathrm{mmol})$ in freshly distilled $\mathrm{Et}_{3} \mathrm{~N}(28 \mathrm{~mL})$ was successively added the corresponding terminal alkyne $\mathbf{8}$ (8.4 mmol), $\mathrm{CuI}$ (0.0133 g, $0.07 \mathrm{mmol})$ and $\mathrm{Pd}\left(\mathrm{PPh}_{3}\right)_{4}$ $(0.161 \mathrm{~g}, 0.14 \mathrm{mmol})$. The resulting mixture was stirred at $50{ }^{\circ} \mathrm{C}$ for $5 \mathrm{~h}$ and after that, a white solid was filtered off through a short plug of celite, washed with ethyl acetate $(3 \times 10 \mathrm{~mL})$ and concentrated (15 Torr). The residue was purified by column chromatography (hexane/ethyl acetate) to yield pure compounds 9. Yields for these compounds 9 are given on Table I. Physical and spectroscopic data follow.

2-(Trimethylsilylethynyl)benzaldehyde (9a): yellow solid; mp $42-43{ }^{\circ} \mathrm{C}$ (hexane $\left./ \mathrm{CH}_{2} \mathrm{Cl}_{2}\right) ; R_{\mathrm{F}}$ 0.58 (hexane/AcOEt 20:1); IR (KBr) v 2957, 2854, 2761, 2151, 1697, 1592, 1247, 866, 845, $763 \mathrm{~cm}^{-1}$; ${ }^{1} \mathrm{H}-\mathrm{NMR}\left(400 \mathrm{MHz}, \mathrm{CDCl}_{3}\right) \delta 10.56(1 \mathrm{H}, \mathrm{d}, J=$ $0.9 \mathrm{~Hz}, \mathrm{COH}), 7.93-7.89$ (1H, m, ArH), 7.60-7.51 $(1 \mathrm{H}, \mathrm{m}, \mathrm{ArH}), 7.46-7.41$ (1H, m, ArH), $0.28[9 \mathrm{H}$, s, $\left.\mathrm{Si}\left(\mathrm{CH}_{3}\right)_{3}\right] ;{ }^{13} \mathrm{C}-\mathrm{NMR}\left(101 \mathrm{MHz}, \mathrm{CDCl}_{3}\right) \delta 192.0$ $(\mathrm{CH}), 136.3(\mathrm{C}), 133.8(\mathrm{CH}), 133.6(\mathrm{CH}), 129.0$ (C), $127.0(\mathrm{C}), 126.9(\mathrm{CH}), 102.5(\mathrm{C}), 100.2(\mathrm{C})$, $0.1\left(\mathrm{CH}_{3}\right)$; LRMS (EI) m/z $202\left(\mathrm{M}^{+}, 4 \%\right), 201$ (18), 188 (18), 187(100), 161 (11), 128 (27). 
2-(Oct-1-yn-1-yl)benzaldehyde (9b): colourless oil; $R_{\mathrm{F}} 0.62$ (hexane/AcOEt 10:1); IR (film) v 2957, 2929, 2856, 1697, 1594, 1192, 823, 761, $726637 \mathrm{~cm}^{-1}$; ${ }^{1} \mathrm{H}-\mathrm{NMR}$ (400 MHz, $\mathrm{CDCl}_{3}$ ) $\delta 10.54(1 \mathrm{H}, \mathrm{d}, J=0.8 \mathrm{~Hz}, \mathrm{COH}), 7.88(1 \mathrm{H}, \mathrm{dt}$, $J=7.9,1.0 \mathrm{~Hz}, \mathrm{ArH}), 7.55-7.45(2 \mathrm{H}, \mathrm{m}, \mathrm{ArH})$, 7.37 (1H, dddd, $J=7.8,5.8,2.8,0.9 \mathrm{~Hz}, \mathrm{ArH}$ ), $2.48\left(2 \mathrm{H}, \mathrm{t}, J=7.1 \mathrm{~Hz}, \mathrm{CH}_{2}\right), 1.71-1.54(2 \mathrm{H}, \mathrm{m}$, $\left.\mathrm{CH}_{2}\right), 1.52-1.38\left(2 \mathrm{H}, \mathrm{m}, \mathrm{CH}_{2}\right), 1.39-1.26(4 \mathrm{H}, \mathrm{m}$, $\left.2 \times \mathrm{CH}_{2}\right), 0.91\left(3 \mathrm{H}, \mathrm{t}, J=7.0 \mathrm{~Hz}, \mathrm{CH}_{3}\right) ;{ }^{13} \mathrm{C}-\mathrm{NMR}$ $\left(101 \mathrm{MHz}, \mathrm{CDCl}_{3}\right) \delta 192.2(\mathrm{CH}), 135.9(\mathrm{C}), 133.6$, $133.2(\mathrm{CH}), 127.9(\mathrm{C}), 127.8,126.8(\mathrm{CH}), 98.2$, 76.3 (C), 31.3, 28.6, 28.5, 22.5, $19.6\left(\mathrm{CH}_{2}\right), 14.0$ $\left(\mathrm{CH}_{3}\right)$; LRMS (EI) m/z $214\left(\mathrm{M}^{+}, 8 \%\right), 185$ (12), 167 (11), 157 (40), 145 (25), 144 (100), 143 (15), 131 (13), 129 (18), 128 (27), 116 (28), 115 (63).

2-(Phenylethynyl)benzaldehyde (9c): yellow oil; $R_{\mathrm{F}} 0.47$ (hexane/AcOEt 10:1); IR (film) v 3060, 2843, 1695, 1591, 1492, 1264, 1192, 817, 753, 688, $637 \mathrm{~cm}^{-1} ;{ }^{1} \mathrm{H}-\mathrm{NMR}\left(400 \mathrm{MHz}, \mathrm{CDCl}_{3}\right) \delta 10.65(1 \mathrm{H}$, d, $J=0.8 \mathrm{~Hz}, \mathrm{COH}), 7.94(1 \mathrm{H}$, ddd, $J=7.8,1.4,0.6$ $\mathrm{Hz}, \mathrm{ArH}), 7.65-7.61$ (1H, m, ArH), 7.60-7.52 (3H, $\mathrm{m}, \mathrm{ArH}), 7.47-7.41$ (1H, m, ArH), 7.40-7.34 (3H, $\mathrm{m}, \mathrm{ArH}) ;{ }^{13} \mathrm{C}-\mathrm{NMR}\left(101 \mathrm{MHz}, \mathrm{CDCl}_{3}\right) \delta 191.6$ $(\mathrm{CH}), 135.8(\mathrm{C}), 133.7,133.2,131.6,129.0,128.5$, 128.4, 127.2 (CH), 126.8, 122.3, 96.3, $84.8(\mathrm{C})$; LRMS (EI) $m / z 206$ (M+, 60\%), 205 (27), 180 (11), 178 (30), 177 (26), 176 (26), 167 (16), 165 (12), 152 (18), 151 (18), 150 (14), 149 (50), 137 (11), 135 (10), 127 (13), 125 (16), 123 (17), 111 (27), 109 (25), 83 (36), 77 (14), 71 (49), 57 (68), 44 (44), 43 (100).

5-Methoxy-2-(phenylethynyl)benzaldehyde (9d): yellow solid; mp $77-79{ }^{\circ} \mathrm{C}$ (hexane $/ \mathrm{CH}_{2} \mathrm{Cl}_{2}$ ); $R_{\mathrm{F}} 0.38$ (hexane/AcOEt 10:1); IR (KBr) $v 2848$, $1685,1604,1594,1499,1323,1226,1164,1023$, 830, 753, 746, 684, $619 \mathrm{~cm}^{-1}$; ${ }^{1} \mathrm{H}-\mathrm{NMR}(400 \mathrm{MHz}$, $\left.\mathrm{CDCl}_{3}\right) \delta 10.61(1 \mathrm{H}, \mathrm{s}, \mathrm{COH}), 7.58-7.50(3 \mathrm{H}, \mathrm{m}$, ArH), $7.42(1 \mathrm{H}, \mathrm{d}, J=2.8 \mathrm{~Hz}, \mathrm{ArH}), 7.38-7.33$ $(3 \mathrm{H}, \mathrm{m}, \mathrm{ArH}), 7.13(1 \mathrm{H}, \mathrm{dd}, J=8.6,2.8 \mathrm{~Hz}, \mathrm{ArH})$, $3.87\left(3 \mathrm{H}, \mathrm{s}, \mathrm{CH}_{3}\right) ;{ }^{13} \mathrm{C}-\mathrm{NMR}\left(101 \mathrm{MHz}, \mathrm{CDCl}_{3}\right) \delta$ $191.5(\mathrm{CH}), 159.7,137.1(\mathrm{C}), 134.5,131.4,128.7$,
$128.4(\mathrm{CH}), 122.6(\mathrm{C}), 121.7(\mathrm{CH}), 119.6(\mathrm{C})$, $109.8(\mathrm{CH}), 94.8,84.8(\mathrm{C}), 55.6\left(\mathrm{CH}_{3}\right)$; LRMS (EI) $\mathrm{m} / z 236\left(\mathrm{M}^{+}, 100 \%\right), 222$ (13), 221 (67), 193 (27), 165 (63), 164 (17), 163 (19), 149 (24), 139 (11), 70 (15), 43 (65).

\section{2-[(4-Methoxyphenyl)ethynyl]benzaldehyde} (9e): yellow solid; mp $44-46{ }^{\circ} \mathrm{C}$ (hexane $/ \mathrm{CH}_{2} \mathrm{Cl}_{2}$ ); $R_{\mathrm{F}} 0.32$ (hexane/AcOEt 10:1); IR (KBr) v 2843, 1695, 1603, 1591, 1507, 1248, 1018, 839, 766, 635 $\mathrm{cm}^{-1}$; ${ }^{1} \mathrm{H}-\mathrm{NMR}\left(400 \mathrm{MHz}, \mathrm{CDCl}_{3}\right) \delta 10.64(1 \mathrm{H}, \mathrm{d}$, $J=0.8 \mathrm{~Hz}, \mathrm{COH}), 7.93(1 \mathrm{H}, \mathrm{ddd}, J=7.8,1.5,0.6$ $\mathrm{Hz}, \mathrm{ArH}), 7.61(1 \mathrm{H}, \mathrm{ddd}, J=7.8,1.40 .6 \mathrm{~Hz}, \mathrm{ArH})$, $7.56(1 \mathrm{H}, \mathrm{td}, J=7.5,1.4 \mathrm{~Hz}, \mathrm{ArH}), 7.50(2 \mathrm{H}, \mathrm{d}, J$ $=8.9 \mathrm{~Hz}, \mathrm{ArH}), 7.46-7.37(1 \mathrm{H}, \mathrm{m}, \mathrm{ArH}), 6.90(2 \mathrm{H}$, $\mathrm{d}, J=8.8 \mathrm{~Hz}, \mathrm{ArH}), 3.83\left(3 \mathrm{H}, \mathrm{s}, \mathrm{CH}_{3}\right) ;{ }^{13} \mathrm{C}-\mathrm{NMR}$ $\left(101 \mathrm{MHz}, \mathrm{CDCl}_{3}\right) \delta 191.8(\mathrm{CH}), 160.2,135.6(\mathrm{C})$, 133.7, 133.2, 133.0, $128.2(\mathrm{CH}), 127.3(\mathrm{C}), 127.2$ $(\mathrm{CH}), 114.3(\mathrm{C}), 114.2(\mathrm{CH}), 96.5,83.7(\mathrm{C}), 55.3$ $\left(\mathrm{CH}_{3}\right)$; LRMS (EI) m/z $236\left(\mathrm{M}^{+}, 100 \%\right), 221$ (61), 193 (19), 165 (57), 164 (15), 163 (17).

SYNTHESIS OF 2-[2-(OCT-1-YL)PHENYL] ACETALDEHYDE (13)

A $1 \mathrm{M}$ solution of potassium hexamethyldisilazide (KHMDS, 2.0 mL, 2.0 mmol) was added to a suspension of (methoxymethyl) triphenylphosphonium chloride $(0.684 \mathrm{~g}, 2.0 \mathrm{mmol})$ in THF $(4 \mathrm{~mL})$ at $-78^{\circ} \mathrm{C}$. The reaction mixture was stirred at the same temperature for $1 \mathrm{~h}$. After that, a solution of aldehyde $9 \mathbf{b}(0.214$ $\mathrm{g}, 1.0 \mathrm{mmol})$ in THF $(8 \mathrm{~mL})$ and stirring was maintained for $3 \mathrm{~h}$ allowing the system to reach $0{ }^{\circ} \mathrm{C}$. The resulting mixture was filtered through a short plug of celite, washed with hexane $(3 \times 15$ $\mathrm{mL}$ ) and concentrated (15 Torr). The residue was dissolved in THF $(1.5 \mathrm{~mL})$, treated with a $3 \mathrm{M}$ solution of $\mathrm{HCl}(0.25 \mathrm{~mL}, 0.75 \mathrm{mmol})$ and the resulting solution was stirred at $78{ }^{\circ} \mathrm{C}$ for $18 \mathrm{~h}$. After that, the reaction mixture was cooled down to room temperature, basified with a saturated sodium bicarbonate aqueous solution and extracted with ethyl acetate $(3 \times 15 \mathrm{~mL})$. The organic layer was washed with brine $(2 \times 10 \mathrm{~mL})$, dried over 
anhydrous magnesium sulfate and evaporated (15 Torr) to yield aldehyde $\mathbf{1 6}$ which was used in the next step without purification.

GENERAL PROCEDURE FOR THE PREPARATION OF N-TERT-BUTANESULFINYL ALDIMINES 4A AND 14

To a solution of $(R)$-tert-butanesulfinamide (1, $0.601 \mathrm{~g}, 5.0 \mathrm{mmol})$ and the corresponding aldehyde $(5.5 \mathrm{mmol})$ in THF $(20 \mathrm{~mL})$ was added Ti(OEt) $(2.28 \mathrm{~g}, 2.08 \mathrm{~mL}, 10.0 \mathrm{mmol})$ at room temperature under argon. The resulting mixture was stirred for 5 $\mathrm{h}$ at the same temperature, and after that, quenched with brine $(3.0 \mathrm{~mL})$, and diluted with ethyl acetate $(50.0 \mathrm{~mL})$. The resulting suspension was filtered through a short plug of Celite and concentrated Then, the reaction mixture was filtered through a short plug of Celite and the solvent was evaporated (15 Torr). The residue was purified by column chromatography (hexane/EtOAc) to yield pure compounds 4a and 14. Yields for compounds $4 \mathbf{a}$ and $\mathbf{1 4}$ are given in Figures 2 and 4, respectively. Physical and spectroscopic data follow.

$\left(\mathrm{R}_{S}\right)$ - N-(tert-Butanesulfinyl) - N-(2vinylbenzyliden)amine (4a): yellow oil; $R_{\mathrm{F}} 0.32$ (hexane/AcOEt 9:1); =-166.5 (c 1.05, $\left.\mathrm{CH}_{2} \mathrm{Cl}_{2}\right)$; IR (film) v 3060, 2981, 2962, 2932, 2864, 1602, 1584 , 1474, 1363, 1221, 1183, 1082, 983, 920, 769, 715 $\mathrm{cm}^{-1}$; ${ }^{1} \mathrm{H}-\mathrm{NMR}\left(400 \mathrm{MHz}, \mathrm{CDCl}_{3}\right) \delta 8.93(1 \mathrm{H}, \mathrm{s}$, $\mathrm{CNH}), 7.95(1 \mathrm{H}, \mathrm{dd}, J=7.8,1.5 \mathrm{~Hz}, \mathrm{ArH}), 7.55$ $(1 \mathrm{H}, \mathrm{dd}, J=7.7,1.4 \mathrm{~Hz}, \mathrm{ArH}), 7.48(1 \mathrm{H}, \mathrm{td}, J=7.6$, $1.5 \mathrm{~Hz}, \mathrm{ArH}), 7.43-7.32\left(2 \mathrm{H}, \mathrm{m}, \mathrm{ArH}, \mathrm{CH}=\mathrm{CH}_{2}\right.$ ), $5.67(1 \mathrm{H}, \mathrm{dd}, J=17.3,1.2 \mathrm{~Hz}, \mathrm{CH}=\mathrm{CH} H), 5.47$ $(1 \mathrm{H}, \mathrm{dd}, J=11.0,1.2 \mathrm{~Hz}, \mathrm{CH}=\mathrm{CHH}), 1.28[9 \mathrm{H}, \mathrm{s}$, $\left.\mathrm{C}\left(\mathrm{CH}_{3}\right)_{3}\right] ;{ }^{13} \mathrm{C}$-NMR $161.3(\mathrm{CH}), 139.7(\mathrm{C}), 133.6$, $132.1(\mathrm{CH}), 131.0(\mathrm{C}), 129.1,127.9,127.3(\mathrm{CH})$, $118.9\left(\mathrm{CH}_{2}\right), 57.7(\mathrm{C}), 22.6\left(\mathrm{CH}_{3}\right)$; LRMS (EI) $\mathrm{m} / \mathrm{z}$ $235\left(\mathrm{M}^{+}, 1 \%\right), 179$ (21), 149 (16), 132 (12), 131 (18), 130 (51), 116 (30), 115 (10), 77 (11), 70 (19), 61 (16), 57 (52), 45 (17), 43 (100), 41 (16).

$\left(\mathrm{R}_{S}\right)-\mathrm{N}-($ tert-Butanesulfinyl $)-\mathrm{N}-\{2-[2-(1-$ octynyl)phenyl]ethyliden $\}$ amine (14): yellow oil; $R_{\mathrm{F}} 0.31$ (hexane/AcOEt 9:1); = -186.0 (c 1.05,
$\mathrm{CH}_{2} \mathrm{Cl}_{2}$ ); IR (film) v 2955, 2928, 2858, 1697, 1619, 1457, 1087, $756 \mathrm{~cm}^{-1}$; ${ }^{1} \mathrm{H}-\mathrm{NMR}\left(400 \mathrm{MHz}, \mathrm{CDCl}_{3}\right.$ ) $\delta 8.14(1 \mathrm{H}, \mathrm{t}, J=4.9 \mathrm{~Hz}, \mathrm{CNH}), 7.45-7.39(1 \mathrm{H}, \mathrm{m}$, ArH), 7.26-7.15 (3H, m, ArH), 4.00 (2H, t, $J=4.8$ $\left.\mathrm{Hz}, \mathrm{CH}_{2}\right), 2.42\left(2 \mathrm{H}, \mathrm{t}, J=7.1 \mathrm{~Hz}, \mathrm{CH}_{2}\right), 1.67-1.53$ $\left(2 \mathrm{H}, \mathrm{m}, \mathrm{CH}_{2}\right), 1.51-1.41\left(2 \mathrm{H}, \mathrm{m}, \mathrm{CH}_{2}\right), 1.37-1.27$ $\left(4 \mathrm{H}, \mathrm{m}, 2 \times \mathrm{CH}_{2}\right), 1.17$ [9H, s, $\left.\mathrm{C}\left(\mathrm{CH}_{3}\right)\right], 0.95-0.84$ $\left(3 \mathrm{H}, \mathrm{m}, \mathrm{CH}_{3}\right) ;{ }^{13} \mathrm{C}-\mathrm{NMR} 167.3(\mathrm{CH}), 136.7(\mathrm{C})$, 132.4, 129.4, 127.8, $127.0(\mathrm{CH}), 124.3,95.4,78.8$, 56.8 (C), 41.3, 31.3, 28.7, $28.622 .5\left(\mathrm{CH}_{2}\right), 22.4$ $\left(\mathrm{CH}_{3}\right), 19.5\left(\mathrm{CH}_{2}\right), 14.0\left(\mathrm{CH}_{3}\right)$; LRMS (EI) $\mathrm{m} / \mathrm{z}$ $275\left(\mathrm{M}^{+} \mathrm{C}_{4} \mathrm{H}_{8}, 5 \%\right), 230$ (11), 229 (62), 227 (11), 226 (37), 170 (15), 169 (16), 168 (13), 167 (15), 157 (22), 156 (27), 155 (22), 154 (14), 143 (19), 142 (14), 141 (22), 131 (14), 130 (20), 129 (29), 128 (37), 127 (18), 116 (12), 115 (30), 113 (22), 85 (16), 57 (100), 55 (20), 43 (51), 41 (30).

SYNTHESIS OF $\left(1 S, R_{\mathrm{S}}\right)$-N-(TERTBUTANESULFINYL)-1-(2-VINYLPHENYL)-4(TRIMETHYLSILYL)BUT-3-YN-1-AMINE (6A)

A mixture of imine 4a $(0.118 \mathrm{~g}, 0.5 \mathrm{mmol})$, 3-bromo1-trimethylsilyl-1-propyne (5; $313 \mathrm{mg}, 0.275 \mathrm{~mL}$, $1.65 \mathrm{mmol}$ ), and indium (189 mg, $1.65 \mathrm{mmol}$ ) was sonicated in dry THF $(2 \mathrm{~mL})$ for $7 \mathrm{~h}$. Then the resulting mixture was hydrolyzed with $\mathrm{H}_{2} \mathrm{O}(5$ $\mathrm{mL})$ and extracted with ethyl acetate $(3 \times 15 \mathrm{~mL})$. The organic phase was washed with brine $(3 \times 10$ $\mathrm{mL})$, dried with anhydrous magnesium sulfate, and the solvent evaporated (15 Torr). The residue was purified by column chromatography (silica gel, hexane/EtOAc) to yield pure compound $\mathbf{6 a}$ as a pale yellow oil (0.082 $\mathrm{g}, 0.35 \mathrm{mmol}, 70 \%): R_{\mathrm{F}} 0.50$ (hexane/AcOEt 2:1); =-78.3 (c 2.24, $\mathrm{CH}_{2} \mathrm{Cl}_{2}$ ); IR (film) v 3193, 2958, 2897, 2868, 2174, 1474, 1411, $1363,1249,1053,840,759 \mathrm{~cm}^{-1}$; ${ }^{1} \mathrm{H}-\mathrm{NMR}(300$ $\left.\mathrm{MHz}, \mathrm{CDCl}_{3}\right) \delta$ 7.48-7.43 (1H, m, ArH), 7.42-7.37 (1H, m, ArH), 7.31-7.25 (2H, m, ArH), $7.13(1 \mathrm{H}$, $\left.\mathrm{dd}, J=17.2,10.9 \mathrm{~Hz}, \mathrm{CH}=\mathrm{CH}_{2}\right), 5.61(1 \mathrm{H}, \mathrm{dd}, J$ $=17.2,1.5 \mathrm{~Hz}, \mathrm{CH}=\mathrm{CH} H), 5.36(1 \mathrm{H}, \mathrm{dd}, J=10.9$, $1.5 \mathrm{~Hz}, \mathrm{CH}=\mathrm{CHH}), 4.89(1 \mathrm{H}, \mathrm{ddd}, J=8.2,5.1,3.0$ $\mathrm{Hz}, \mathrm{CH}), 4.23(1 \mathrm{H}, \mathrm{d}, J=3.0 \mathrm{~Hz}, \mathrm{NH}), 2.73(1 \mathrm{H}$, 
dd, $J=16.9,5.1 \mathrm{~Hz}, \mathrm{CH} H), 2.63(1 \mathrm{H}, \mathrm{dd}, J=16.9$, $8.4 \mathrm{~Hz}, \mathrm{CHH}), 1.24\left[9 \mathrm{H}, \mathrm{s}, \mathrm{C}\left(\mathrm{CH}_{3}\right)_{3}\right], 0.16[9 \mathrm{H}$, s, $\left.\mathrm{Si}\left(\mathrm{CH}_{3}\right)_{3}\right] ;{ }^{13} \mathrm{C}-\mathrm{NMR}\left(75 \mathrm{MHz}, \mathrm{CDCl}_{3}\right) \delta 137.3$, $137.2(\mathrm{C}), 134.1,128.0,127.7,127.2,126.8(\mathrm{CH})$, $117.5\left(\mathrm{CH}_{2}\right), 102.1,86.2,55.8(\mathrm{C}), 52.4(\mathrm{CH})$, $29.2\left(\mathrm{CH}_{2}\right), 22.6,-0.08\left(\mathrm{CH}_{3}\right)$; LRMS (EI) $\mathrm{m} / \mathrm{z} 243$ $\left(\mathrm{M}^{+} \mathrm{C}_{4} \mathrm{H}_{8} \mathrm{OS}, 4 \%\right), 228$ (11), 207 (13), 180 (25), 179 (19), 167 (16), 165 (12), 164 (100), 132 (37), 131 (25), 130 (66), 128 (15), 117 (23), 116 (39), 115 (26), 77 (15), 73(76).

GENERAL PROCEDURE FOR THE AMINOALLYLATION OF ALDEHYDES 9: SYNTHESIS OF ENYNES 11

A mixture of indium powder (173 mg, $1.50 \mathrm{mmol})$, $\left(R_{\mathrm{S}}\right)$-N-tert-butanesulfinamide $(\mathbf{1}, 0.121 \mathrm{~g}, 1.0$ $\mathrm{mmol})$, the corresponding aldehyde $\mathbf{9}(1.15 \mathrm{mmol})$, and $\mathrm{Ti}(\mathrm{OEt})_{4}(0.456 \mathrm{~g}, 0.450 \mathrm{~mL}, 2.0 \mathrm{mmol})$ in THF $(2 \mathrm{~mL})$ was stirred under argon for $1 \mathrm{~h}$ at $23{ }^{\circ} \mathrm{C}$. At this time, allyl bromide $(\mathbf{1 0}, 0.254 \mathrm{~g}, 0.18 \mathrm{~mL}, 1.50$ $\mathrm{mmol}$ ) was added and the reaction mixture heated for $5 \mathrm{~h}$ at $60^{\circ} \mathrm{C}$. The mixture was allowed to cool to room temperature, quenched with brine $(2 \mathrm{~mL})$, and diluted with ethyl acetate. The resulting suspension was filtered through a short plug of Celite and the solvent evaporated (15 Torr). The residue was purified by column chromatography (hexane/ EtOAc) to yield pure compounds 11. Yields are given on Table I. Physical and spectroscopic data follow.

(1 S, $\left.\mathrm{R}_{S}\right)-\mathrm{N}-($ tert-Butanesulfinyl) - 1- [2(trimethylsilylethynyl)phenyl]but-3-en-1-amine (11a): colourless oil; $R_{\mathrm{F}} 0.38$ (hexane/AcOEt 2:1); $=-68.0\left(\right.$ c 1.10, $\left.\mathrm{CH}_{2} \mathrm{Cl}_{2}\right)$; IR (film) v 3075, 2958, 2154, 1475, 1447, 1249, 1053, 862, 840, 758, 644 $\mathrm{cm}^{-1} ;{ }^{1} \mathrm{H}-\mathrm{NMR}\left(300 \mathrm{MHz}, \mathrm{CDCl}_{3}\right) \delta 7.46(1 \mathrm{H}$, ddd, $J=7.5,1.4,0.6 \mathrm{~Hz}, \mathrm{ArH}), 7.38-7.26$ (2H, m, ArH), $7.20(1 \mathrm{H}, \mathrm{ddd}, J=7.6,6.9,2.0 \mathrm{~Hz}, \mathrm{ArH})$, 5.87-5.60 (1H, m, $\left.\mathrm{CH}=\mathrm{CH}_{2}\right), 5.23-5.11(2 \mathrm{H}, \mathrm{m}$, $\left.\mathrm{CH}=\mathrm{CH}_{2}\right), 5.02-4.90(1 \mathrm{H}, \mathrm{m}, \mathrm{CH}), 3.80(1 \mathrm{H}, \mathrm{d}, J$ $=3.9 \mathrm{~Hz}, \mathrm{NH}), 2.84-2.69(1 \mathrm{H}, \mathrm{m}, \mathrm{CH} H), 2.63-2.46$ $(1 \mathrm{H}, \mathrm{m}, \mathrm{CHH}), 1.21\left[9 \mathrm{H}, \mathrm{s}, \mathrm{C}\left(\mathrm{CH}_{3}\right)_{3}\right], 0.27[9 \mathrm{H}$, s, $\left.\mathrm{Si}\left(\mathrm{CH}_{3}\right)_{3}\right] ;{ }^{13} \mathrm{C}-\mathrm{NMR}\left(75 \mathrm{MHz}, \mathrm{CDCl}_{3}\right) \delta 144.2$ (C), 134.3, 133.0, 128.5, 127.0, $126.8(\mathrm{CH}), 121.8$ (C), $119.0\left(\mathrm{CH}_{2}\right), 102.8,100.2(\mathrm{C}), 55.9(\mathrm{CH}), 41.7$ $\left(\mathrm{CH}_{2}\right), 22.6\left(\mathrm{CH}_{3}\right),-0.08\left(\mathrm{CH}_{3}\right)$; LRMS (EI) $\mathrm{m} / \mathrm{z}$ $291\left(\mathrm{M}^{+}-\mathrm{C}_{4} \mathrm{H}_{8}, 1 \%\right), 235$ (17), 234 (100), 231 (17), 230 (24), 218 (54), 202 (25), 200 (21), 186 (32), 160 (20), 156 (97), 143 (23), 75 (21), 73 (75), 59 (16).

$\left(1 \mathrm{~S}, \mathrm{R}_{S}\right)-\mathrm{N}-($ tert-Butanesulfinyl)-1-[2-(oct-1yn-1-yl)phenyl]but-3-en-1-amine (11b): colourless oil; $R_{\mathrm{F}} 0.43$ (hexane/AcOEt 2:1); $=-59.3$ (c 1.12, $\mathrm{CH}_{2} \mathrm{Cl}_{2}$ ); IR (film) v 3065, 2955, 2929, 2858, 1638, 1448, 1362, 1055, 910, $757 \mathrm{~cm}^{-1}$; ${ }^{1} \mathrm{H}-\mathrm{NMR}$ (300 $\left.\mathrm{MHz}, \mathrm{CDCl}_{3}\right) \delta 7.40(1 \mathrm{H}, \mathrm{dd}, J=7.6,1.5 \mathrm{~Hz}, \mathrm{ArH})$, $7.31(1 \mathrm{H}, \mathrm{dd}, J=7.7,1.7 \mathrm{~Hz}, \operatorname{ArH}), 7.23(1 \mathrm{H}, \mathrm{dd}$, $J=7.6,1.6 \mathrm{~Hz}, \mathrm{ArH}), 7.18(1 \mathrm{H}, \mathrm{td}, J=7.3,1.7 \mathrm{~Hz}$, ArH), 5.83-5.64 (1H, m, $\left.\mathrm{CH}=\mathrm{CH}_{2}\right), 5.23-5.10(2 \mathrm{H}$, $\left.\mathrm{m}, \mathrm{CH}=\mathrm{CH}_{2}\right), 5.04-4.94(1 \mathrm{H}, \mathrm{m}, \mathrm{CH}), 3.77(1 \mathrm{H}, \mathrm{d}$, $J=3.9 \mathrm{~Hz}, \mathrm{NH}), 2.82-2.66(1 \mathrm{H}, \mathrm{m}, \mathrm{CH} H), 2.61-$ $2.47(1 \mathrm{H}, \mathrm{m}, \mathrm{CHH}), 2.45\left(2 \mathrm{H}, \mathrm{t}, J=7.1 \mathrm{~Hz}, \mathrm{CH}_{2}\right)$, 1.68-1.57 (3H, m, $\left.\mathrm{CH}_{2}, \mathrm{CH}\right), 1.51-1.42(1 \mathrm{H}, \mathrm{m}$, $\mathrm{CHH}), 1.38-1.28\left(4 \mathrm{H}, \mathrm{m}, 2 \times \mathrm{CH}_{2}\right), 1.20(9 \mathrm{H}, \mathrm{s}$, $\left.\mathrm{C}\left(\mathrm{CH}_{3}\right)_{3}\right), 0.96-0.85\left(3 \mathrm{H}, \mathrm{m}, \mathrm{CH}_{3}\right) ;{ }^{13} \mathrm{C}-\mathrm{NMR}(75$ $\left.\mathrm{MHz}, \mathrm{CDCl}_{3}\right) \delta 143.4(\mathrm{C}), 134.3,132.6,127.5$, 126.9, $126.6(\mathrm{CH}), 122.8(\mathrm{C}), 118.9\left(\mathrm{CH}_{2}\right), 96.1$, $55.8(\mathrm{C}), 55.6(\mathrm{CH}), 42.0,31.3,28.7,28.6\left(\mathrm{CH}_{2}\right)$, $22.6\left(\mathrm{CH}_{3}\right), 22.5,19.6\left(\mathrm{CH}_{2}\right), 14.1\left(\mathrm{CH}_{3}\right)$; LRMS (EI) $m / z 287\left(\mathrm{M}^{+}-\mathrm{C}_{5} \mathrm{H}_{12}, 3 \%\right), 262$ (23), 246 (26), 243 (18), 242 (100), 227 (20), 226 (99), 214 (29), 213 (22), 212 (21), 183 (30), 172 (20), 170 (29), 168 (25), 157 (20), 156 (84), 155 (22), 154 (20), 144 (14), 143 (48), 142 (36), 141 (22), 130 (36), 129 (24), 128 (24).

$\left(1 \mathrm{~S}, \mathrm{R}_{S}\right)-\mathrm{N}-($ tert-Butanesulfinyl $)-1-[2-$ (phenylethynyl)phenyl]but-3-en-1-amine (11c): colourless oil; $R_{\mathrm{F}} 0.25$ (hexane/AcOEt 2:1); = -42.9 (c 1.61, $\mathrm{CH}_{2} \mathrm{Cl}_{2}$ ); IR (film) v 3214, 3061, 2960, 2922, 2867, 1638, 1492, 1473, 1261, 1053, 912, 755, $689 \mathrm{~cm}^{-1} ;{ }^{1} \mathrm{H}-\mathrm{NMR}\left(400 \mathrm{MHz}, \mathrm{CDCl}_{3}\right) \delta$ 7.57-7.51 (3H, m, ArH), 7.41-7.27 (6H, m, ArH), 5.84-5.70 $\left(1 \mathrm{H}, \mathrm{m}, \mathrm{CH}=\mathrm{CH}_{2}\right), 5.23-5.13(2 \mathrm{H}, \mathrm{m}$, $\left.\mathrm{CH}=\mathrm{CH}_{2}\right), 5.12-5.04(1 \mathrm{H}, \mathrm{m}, \mathrm{CH}), 3.80(1 \mathrm{H}, \mathrm{d}, J=$ 
$3.7 \mathrm{~Hz}, \mathrm{NH}), 2.81(1 \mathrm{H}, \mathrm{dt}, J=14.1,5.9 \mathrm{~Hz}, \mathrm{CH} H)$, $2.62(1 \mathrm{H}, \mathrm{dt}, J=14.8,7.9 \mathrm{~Hz}, \mathrm{CHH}), 1.21[9 \mathrm{H}, \mathrm{s}$, $\left.\mathrm{C}\left(\mathrm{CH}_{3}\right)_{3}\right] ;{ }^{13} \mathrm{C}-\mathrm{NMR}\left(75 \mathrm{MHz}, \mathrm{CDCl}_{3}\right) \delta 143.6(\mathrm{C})$, 134.2, 132.6, 131.5, 128.5, 128.4, 128.3, 127.1, $126.9(\mathrm{CH}), 123.0,122.1(\mathrm{C}), 119.1\left(\mathrm{CH}_{2}\right), 94.7$, 87.1, $55.8(\mathrm{C}), 55.7(\mathrm{CH}), 42.0\left(\mathrm{CH}_{2}\right), 22.6\left(\mathrm{CH}_{3}\right)$; LRMS (EI) $m / z 247\left(\mathrm{M}^{+}-\mathrm{C}_{4} \mathrm{H}_{8} \mathrm{SO}, 6 \%\right), 237$ (15), 236 (17), 235 (24), 234 (100), 219 (18), 218 (22), 207 (23), 205 (57), 204 (69), 203 (19), 202 (23), 176 (18).

(1S, $\left.\mathrm{R}_{S}\right)-\mathrm{N}-($ tert-Butanesulfinyl)-1-[5-methoxy2-(phenylethynyl)phenyl]but-3-en-1-amine (11d): colourless oil; $R_{\mathrm{F}} 0.23$ (hexane/AcOEt 2:1); $=-41.8$ (c 2.08, $\mathrm{CH}_{2} \mathrm{Cl}_{2}$ ); IR (film) v 3075, 2951, 2834, 1732, 1606, 1595, 1498, 1292, 1233, 1032, 914, $821,755,690 \mathrm{~cm}^{-1} ;{ }^{1} \mathrm{H}-\mathrm{NMR}\left(300 \mathrm{MHz}, \mathrm{CDCl}_{3}\right) \delta$ 7.54-7.45 (3H, m, ArH), 7.37-7.30 (3H, m, ArH), $6.95(1 \mathrm{H}, \mathrm{d}, J=2.7 \mathrm{~Hz}, \mathrm{ArH}), 6.79(1 \mathrm{H}, \mathrm{dd}, J=8.5$, $2.6 \mathrm{~Hz}, \mathrm{ArH}), 5.90-5.68\left(1 \mathrm{H}, \mathrm{m}, \mathrm{CH}=\mathrm{CH}_{2}\right), 5.25-$ $5.13\left(2 \mathrm{H}, \mathrm{m}, \mathrm{CH}=\mathrm{CH}_{2}\right), 5.08-4.99(1 \mathrm{H}, \mathrm{m}, \mathrm{CH})$, $3.82\left(3 \mathrm{H}, \mathrm{s}, \mathrm{CH}_{3}\right), 2.81(1 \mathrm{H}, \mathrm{dt}, J=14.5,6.0 \mathrm{~Hz}$, $\mathrm{CH} H), 2.59$ (1H, dt, $J=14.8,8.0 \mathrm{~Hz}, \mathrm{CHH}), 1.22$ $\left[9 \mathrm{H}, \mathrm{s}, \mathrm{C}\left(\mathrm{CH}_{3}\right)_{3}\right] ;{ }^{13} \mathrm{C}-\mathrm{NMR}\left(75 \mathrm{MHz}, \mathrm{CDCl}_{3}\right) \delta$ 159.7, 145.5 (C), 134.2, 134.0, 131.3, 128.3, 128.1 $(\mathrm{CH}), 123.3(\mathrm{C}), 119.1\left(\mathrm{CH}_{2}\right), 114.1(\mathrm{C}), 112.8$, $112.6(\mathrm{CH}), 93.4,87.1(\mathrm{C}), 55.9(\mathrm{C}), 55.7(\mathrm{CH})$, $55.3\left(\mathrm{CH}_{3}\right), 42.0\left(\mathrm{CH}_{2}\right), 22.6\left(\mathrm{CH}_{3}\right)$; LRMS (EI) $\mathrm{m} / \mathrm{z} 277\left(\mathrm{M}^{+}-\mathrm{C}_{4} \mathrm{H}_{8} \mathrm{OS}, 10 \%\right), 237$ (25), 236 (100), 235 (18), 215 (12), 192 (13), 191 (13), 57 (20).

$\left(1 \mathrm{~S}, \mathrm{R}_{S}\right)-\mathrm{N}-($ tert-Butanesulfinyl $)-1-\{2-[(4-$ methoxyphenyl)ethynyl]phenyl $\}$ but-3-en-1-amine (11e): colourless oil; $R_{\mathrm{F}} 0.19$ (hexane/AcOEt $2: 1)$; $=-22.2\left(\right.$ c $1.38, \mathrm{CH}_{2} \mathrm{Cl}_{2}$ ); IR (film) v 3213, 3065, 2976, 2957, 2908, 2209, 1605, 1509, 1459, 1286, 1248, 1174, 1054, 1030, 913, 831, $758 \mathrm{~cm}^{-1}$; ${ }^{1} \mathrm{H}-\mathrm{NMR}\left(400 \mathrm{MHz}, \mathrm{CDCl}_{3}\right) \delta$ 7.53-7.45 $(3 \mathrm{H}, \mathrm{m}$, ArH), $7.37(1 \mathrm{H}, \mathrm{dd}, J=7.7,1.5 \mathrm{~Hz}, \mathrm{ArH}), 7.30$ $(1 \mathrm{H}, \mathrm{td}, J=7.5,1.5 \mathrm{~Hz}, \mathrm{ArH}), 7.26-7.21(1 \mathrm{H}, \mathrm{m}$, ArH), 6.91-6.85 (2H, m, ArH), 5.87-5.69 (1H, $\left.\mathrm{m}, \mathrm{CH}=\mathrm{CH}_{2}\right), 5.24-5.13\left(2 \mathrm{H}, \mathrm{m}, \mathrm{CH}=\mathrm{CH}_{2}\right), 5.11-$ $5.02(1 \mathrm{H}, \mathrm{m}, \mathrm{CH}), 3.83\left(4 \mathrm{H}, \mathrm{s}, \mathrm{CH}_{3}, \mathrm{NH}\right), 2.84-$ $2.76(1 \mathrm{H}, \mathrm{m}, \mathrm{CH} H), 2.62(1 \mathrm{H}, \mathrm{dt}, J=14.8,7.9 \mathrm{~Hz}$,
$\mathrm{CHH}), 1.20\left[9 \mathrm{H}, \mathrm{s}, \mathrm{C}\left(\mathrm{CH}_{3}\right)_{3}\right] ;{ }^{13} \mathrm{C}-\mathrm{NMR}(101 \mathrm{MHz}$, $\left.\mathrm{CDCl}_{3}\right) \delta 159.7,143.3(\mathrm{C}), 134.3,133.0,132.4$, 128.0, 127.1, $126.8(\mathrm{CH}), 122.4(\mathrm{C}), 119.0\left(\mathrm{CH}_{2}\right)$, $115.1(\mathrm{C}), 114.0(\mathrm{CH}), 94.8,85.8,(\mathrm{C}), 55.8(\mathrm{CH})$, $55.3\left(\mathrm{CH}_{3}\right), 42.0\left(\mathrm{CH}_{2}\right), 22.7\left(\mathrm{CH}_{3}\right)$; LRMS (EI) $\mathrm{m} / z 277\left(\mathrm{M}^{+}-\mathrm{C}_{4} \mathrm{H}_{8} \mathrm{OS}, 24 \%\right), 276$ (84), 246 (13), 237 (17), 236 (100), 235 (34), 215 (14), 57 (18).

ALLYLATION OF IMINE 14: SYNTHESIS OF HOMOALLYL AMINE DERIVATIVE 15

A mixture of imine 14 (0.166 g, $0.5 \mathrm{mmol})$, allyl bromide $(\mathbf{1 0} ; 0.127 \mathrm{~g}, 0.090 \mathrm{~mL}, 0.75 \mathrm{mmol})$ and indium $(0.063 \mathrm{~g}, 0.55 \mathrm{mmol})$ was stirred in THF $(2 \mathrm{~mL})$ for $6 \mathrm{~h}$ at $60{ }^{\circ} \mathrm{C}$. Then the resulting mixture was hydrolyzed with $\mathrm{H}_{2} \mathrm{O}(5 \mathrm{~mL})$ and extracted with ethyl acetate $(3 \times 15 \mathrm{~mL})$. The organic phase was washed with brine $(3 \times 10 \mathrm{~mL})$, dried with anhydrous magnesium sulfate, and the solvent evaporated (15 Torr). The residue was purified by column chromatography (silica gel, hexane/ EtOAc) to yield pure compound $\mathbf{1 5}$ as a colourless oil $(0.093 \mathrm{~g}, 0.25 \mathrm{mmol}, 50 \%): R_{\mathrm{F}} 0.41$ (hexane/ AcOEt 2:1); =-12.3 (c 1.10, $\mathrm{CH}_{2} \mathrm{Cl}_{2}$ ); IR (film) $v 2954,2928,2858,1638,1448,1363,1051,914$, $756 \mathrm{~cm}^{-1} ;{ }^{1} \mathrm{H}-\mathrm{RMN}\left(400 \mathrm{MHz}, \mathrm{CDCl}_{3}\right) \delta$ 7.47-7.31 (1H, m, ArH), 7.23-7.09 (3H, m, ArH), 5.69-5.75 $\left(1 \mathrm{H}, \mathrm{m}, \mathrm{CH}=\mathrm{CH}_{2}\right), 5.24-5.16\left(2 \mathrm{H}, \mathrm{m}, \mathrm{CH}=\mathrm{CH}_{2}\right)$, 3.77-3.62 (1H, m, CH), $3.37(1 \mathrm{H}, \mathrm{d}, J=7.2 \mathrm{~Hz}$, $\mathrm{NH}), 2.98(1 \mathrm{H}, \mathrm{dd}, J=13.5,8.4 \mathrm{~Hz}, \mathrm{CH} H), 2.92$ $(1 \mathrm{H}, \mathrm{dd}, J=13.5,6.3 \mathrm{~Hz}, \mathrm{CHH}), 2.48-2.39(4 \mathrm{H}$, $\left.\mathrm{m}, 2 \times \mathrm{CH}_{2}\right), 1.68-1.58\left(4 \mathrm{H}, \mathrm{m}, 2 \times \mathrm{CH}_{2}\right), 1.52-$ $1.42\left(4 \mathrm{H}, \mathrm{m}, 2 \times \mathrm{CH}_{2}\right), 1.04\left[9 \mathrm{H}, \mathrm{s}, \mathrm{C}\left(\mathrm{CH}_{3}\right)_{3}\right], 0.95-$ $0.87\left(3 \mathrm{H}, \mathrm{m}, \mathrm{CH}_{3}\right) ;{ }^{13} \mathrm{C}-\mathrm{RMN}\left(101 \mathrm{MHz}, \mathrm{CDCl}_{3}\right) \delta$ $140.4(\mathrm{C}), 134.1,132.2,129.9,127.5,126.2(\mathrm{CH})$, $124.2(\mathrm{C}), 119.3\left(\mathrm{CH}_{2}\right), 94.6,79.4(\mathrm{C}), 56.8(\mathrm{CH})$, 55.8 (C), 40.8, 40.2, 31.4, 28.8, 28.7, $22.6\left(\mathrm{CH}_{2}\right)$, $22.4\left(\mathrm{CH}_{3}\right), 19.6\left(\mathrm{CH}_{2}\right), 14.1\left(\mathrm{CH}_{3}\right)$; LRMS (EI) $m / z 317\left(\mathrm{M}^{+}-\mathrm{C}_{4} \mathrm{H}_{8}, 3 \%\right), 275$ (23), 242 (27), 226 (23), 207 (33), 186 (13), 157 (12), 156 (12), 141 (21), 131 (14), 129 (20), 128 (34), 118 (100), 115 (18), 102 (34). 
GENERAL PROCEDURE FOR THE DESILYLATION OF COMPOUNDS 6A AND 11A: SYNTHESIS OF TERMINAL ALKYNES 6B AND 11F

A suspension of potassium carbonate $(0.005$ $\mathrm{g}, 0.036 \mathrm{mmol})$ in methanol $(4 \mathrm{~mL})$ was added dropwise to a solution of compounds $\mathbf{6 a}$ or 11a $(0.5 \mathrm{mmol})$ in THF $(4 \mathrm{~mL})$. The reaction mixture was stirred for $12 \mathrm{~h}$ at room temperature and then it was hydrolyzed with a $1 \mathrm{M} \mathrm{NH}_{4} \mathrm{Cl}$ aqueous solution $(8 \mathrm{~mL})$ and extracted with methyl tertbutyl ether $(3 \times 15 \mathrm{~mL})$. The organic layer was dried with anhydrous magnesium sulfate and the solvent evaporated (15 Torr). The residue was purified by column chromatography (silica gel, hexane/ EtOAc, 2:1) to yield pure compounds $\mathbf{6 b}$ or $\mathbf{1 1 f}$ in 69 and $>95 \%$ yield, respectively. Physical and spectroscopic data follow.

(1 $\left.\mathrm{S}, \mathrm{R}_{S}\right)-\mathrm{N}-($ tert-Butanesulfinyl)-1-(2vinylphenyl)but-3-yn-1-amine (6b): yellow oil; $R_{\mathrm{F}}$ 0.26 (hexane/AcOEt 2:1); $=-80.9\left(c 1.20, \mathrm{CH}_{2} \mathrm{Cl}_{2}\right)$; IR (film) v 3296, 3218, 2981, 2962, 2922, 2864, 1624, 1474, 1452, 1364, 1053, 916, 762, $637 \mathrm{~cm}^{-1}$; ${ }^{1} \mathrm{H}-\mathrm{NMR}\left(300 \mathrm{MHz}, \mathrm{CDCl}_{3}\right) \delta$ 7.48-7.44 $(1 \mathrm{H}, \mathrm{m}$, ArH), 7.42-7.39 (1H, m, ArH), 7.32-7.27 (2H, m, ArH), $7.12\left(1 \mathrm{H}, \mathrm{dd}, J=17.2,10.9 \mathrm{~Hz}, \mathrm{CH}=\mathrm{CH}_{2}\right)$, $5.62(1 \mathrm{H}, \mathrm{dd}, J=17.2,1.4 \mathrm{~Hz}, \mathrm{CH}=\mathrm{CH} H), 5.37$ $(1 \mathrm{H}, \mathrm{dd}, J=11.0,1.4 \mathrm{~Hz}, \mathrm{CH}=\mathrm{CHH}), 4.92(1 \mathrm{H}$, ddd, $J=8.4,5.1,3.6 \mathrm{~Hz}, \mathrm{CH}), 4.06(1 \mathrm{H}, \mathrm{d}, J=$ $3.6 \mathrm{~Hz}, \mathrm{NH}), 2.74(1 \mathrm{H}$, ddd, $J=16.9,5.2,2.6 \mathrm{~Hz}$, $\mathrm{CH} H), 2.65(1 \mathrm{H}, \mathrm{ddd}, J=16.9,8.1,2.6 \mathrm{~Hz}, \mathrm{CHH})$, $2.12(1 \mathrm{H}, \mathrm{t}, J=2.6 \mathrm{~Hz}, \mathrm{CH}), 1.24\left[9 \mathrm{H}, \mathrm{s}, \mathrm{C}\left(\mathrm{CH}_{3}\right)_{3}\right]$; ${ }^{13} \mathrm{C}-\mathrm{NMR}\left(75 \mathrm{MHz}, \mathrm{CDCl}_{3}\right) \delta 137.3,137.1(\mathrm{C})$, $134.0,128.1,127.8,126.9,126.8(\mathrm{CH}), 117.6$ $\left(\mathrm{CH}_{2}\right), 79.9(\mathrm{C}), 72.2(\mathrm{CH}), 55.9(\mathrm{C}), 52.8(\mathrm{CH})$, $27.7\left(\mathrm{CH}_{2}\right), 22.6\left(\mathrm{CH}_{3}\right)$; LRMS (EI) $\mathrm{m} / z 171\left(\mathrm{M}^{+}-\right.$ $\left.\mathrm{C}_{4} \mathrm{H}_{8} \mathrm{OS}, 2 \%\right), 131$ (12), 130 (100), 128 (11), 116 (19), 115 (19), 77 (10).

(1 S, $\left.\mathrm{R}_{S}\right)-\mathrm{N}-($ tert-Butanesulfinyl)-1-(2ethynylphenyl)but-3-en-1-amine (11f): colourless oil; $R_{\mathrm{F}} 0.22$ (hexane/AcOEt 2:1); = -94.2 (c 1.45, $\mathrm{CH}_{2} \mathrm{Cl}_{2}$ ); IR (film) v 3292, 3203, 2976, 2957, 2868,
1638, 1474, 1446, 1363, 1054, 914, $759 \mathrm{~cm}^{-1}$; ${ }^{1} \mathrm{H}-\mathrm{NMR}\left(400 \mathrm{MHz}, \mathrm{CDCl}_{3}\right) \delta 7.50(1 \mathrm{H}, \mathrm{dd}, J=$ 7.4, 1.1 Hz, ArH), 7.39-7.30 (2H, m, ArH), 7.23 $(1 \mathrm{H}, \mathrm{ddd}, J=7.6,6.9,1.9 \mathrm{~Hz}, \mathrm{ArH}), 5.82-5.68(1 \mathrm{H}$, $\left.\mathrm{m}, \mathrm{CH}=\mathrm{CH}_{2}\right), 5.22-5.14\left(2 \mathrm{H}, \mathrm{m}, \mathrm{CH}=\mathrm{CH}_{2}\right), 5.03$ $(1 \mathrm{H}, \mathrm{ddd}, J=8.5,5.4,3.4 \mathrm{~Hz}, \mathrm{CH}), 3.77(1 \mathrm{H}, \mathrm{d}$, $J=3.5 \mathrm{~Hz}, \mathrm{NH}), 3.35(1 \mathrm{H}, \mathrm{s}, \mathrm{CH}), 2.78-2.67(1 \mathrm{H}$, $\mathrm{m}, \mathrm{CH} H), 2.60-2.46(1 \mathrm{H}, \mathrm{m}, \mathrm{CHH}), 1.20[9 \mathrm{H}, \mathrm{s}$, $\left.\mathrm{C}\left(\mathrm{CH}_{3}\right)_{3}\right] ;{ }^{13} \mathrm{C}-\mathrm{NMR}\left(101 \mathrm{MHz}, \mathrm{CDCl}_{3}\right) \delta(101$ $\left.\mathrm{MHz}, \mathrm{CDCl}_{3}\right) \delta 144.4(\mathrm{C}), 134.1,133.2,128.8$, 127.1, $126.8(\mathrm{CH}), 121.0(\mathrm{C}), 119.2\left(\mathrm{CH}_{2}\right), 82.6$ (C), $81.3(\mathrm{CH}), 55.8(\mathrm{C}), 55.2(\mathrm{CH}), 42.1\left(\mathrm{CH}_{2}\right)$, $22.6\left(\mathrm{CH}_{3}\right)$; LRMS (EI) $\mathrm{m} / z 219\left(\mathrm{M}^{+}-\mathrm{C}_{4} \mathrm{H}_{8}, 3 \%\right)$, 178 (93), 177 (28), 171 (13), 170 (14), 161 (39), 160 (26), 159 (27), 158 (60), 130 (60), 129 (100), 128 (48), 115 (27), 103 (20), 102 (30), 101 (15), 77 (23).

GENERAL PROCEDURE FOR THE RING-CLOSING METATHESIS OF ENYNES 11 AND 15: SYNTHESIS OF DIENES 12 AND 16

A mixture of the corresponding enyne $\mathbf{1 1}$ or $\mathbf{1 5}$ (0.1 mmol), ruthenium Grubbs second generation catalyst $(0.0085 \mathrm{~g}, 0.01 \mathrm{mmol})$ in dry toluene $(10 \mathrm{~mL})$ was stirred at $120{ }^{\circ} \mathrm{C}$ under argon for 1 h. Then the solvent was evaporated (15 Torr) and the resulting residue was purified by column chromatography (silica gel, hexane/AcOEt) to yield pure compounds $\mathbf{1 2}$ and 16. Yields are given on Table II. Physical and spectroscopic data follow.

(1S, $\left.\mathrm{R}_{S}\right)-\mathrm{N}-($ tert-Butanesulfinyl)-4-(oct-1en-2-yl)-1,2-dihydronaphthalen-1-amine (12b): colourless oil; $R_{\mathrm{F}} 0.53$ (hexane/AcOEt 1:1); = -10.3 (c 1.12, $\mathrm{CH}_{2} \mathrm{Cl}_{2}$ ); IR (film) v 3208, 2954, 2926, 2856, 1451, 1362, 1052, 898, 772, $747 \mathrm{~cm}^{-}$ ${ }^{1}$; ${ }^{1} \mathrm{H}-\mathrm{NMR}\left(300 \mathrm{MHz}, \mathrm{CDCl}_{3}\right) \delta$ 7.40-7.36 $(1 \mathrm{H}$, $\mathrm{m}, \operatorname{ArH}), 7.31-7.19$ (3H, m, ArH), $5.86(1 \mathrm{H}, \mathrm{t}, J$ $=4.5 \mathrm{~Hz}, \mathrm{C}=\mathrm{CH}), 5.09(1 \mathrm{H}, \mathrm{dt}, J=2.5,1.3 \mathrm{~Hz}$, $\mathrm{C}=\mathrm{CH} H), 5.00(1 \mathrm{H}, \mathrm{d}, J=2.4 \mathrm{~Hz}, \mathrm{C}=\mathrm{C} H \mathrm{H}), 4.50-$ $4.40(1 \mathrm{H}, \mathrm{m}, \mathrm{CH}), 3.45(1 \mathrm{H}, \mathrm{d}, J=8.8 \mathrm{~Hz}, \mathrm{NH})$, $2.74(1 \mathrm{H}$, ddd, $J=17.0,5.7,4.2 \mathrm{~Hz}, \mathrm{CH} H), 2.58$ $(1 \mathrm{H}, \mathrm{ddd}, J=17.0,7.1,4.9 \mathrm{~Hz}, \mathrm{CHH}), 2.23(2 \mathrm{H}$, 
t, $\left.J=7.3 \mathrm{~Hz}, \mathrm{CH}_{2}\right), 1.40-1.21\left(8 \mathrm{H}, \mathrm{m}, 4 \times \mathrm{CH}_{2}\right)$, $1.19\left[9 \mathrm{H}, \mathrm{s}, \mathrm{C}\left(\mathrm{CH}_{3}\right)_{3}\right], 0.90-0.82\left(3 \mathrm{H}, \mathrm{m}, \mathrm{CH}_{3}\right)$; ${ }^{13} \mathrm{C}-\mathrm{NMR}\left(75 \mathrm{MHz}, \mathrm{CDCl}_{3}\right) \delta 148.7,140.8,136.3$, $133.3(\mathrm{C}), 127.9,127.3,126.6,125.7,122.0(\mathrm{CH})$, $114.1\left(\mathrm{CH}_{2}\right), 56.0(\mathrm{C}), 54.7(\mathrm{CH}), 35.8,32.4,31.7$, 28.9, $28.3\left(\mathrm{CH}_{2}\right), 22.6,14.0\left(\mathrm{CH}_{3}\right)$; LRMS (EI) $\mathrm{m} / \mathrm{z}$ $303\left(\mathrm{M}^{+}-\mathrm{C}_{4} \mathrm{H}_{8}, 4 \%\right), 240$ (25), 239 (100), 238 (38), 169 (14), 168 (19), 167 (27), 155 (36), 154 (13), 153 (20), 152 (11), 142 (10), 141 (67), 129 (13), 57 (31), 43 (11), 41 (13).

(1 S, $\left.\mathrm{R}_{S}\right)-\mathrm{N}-($ tert-Butanesulfinyl)-4-(1phenylvinyl)-1,2-dihydronaphthalen-1-amine (12c): colourless oil; $R_{\mathrm{F}} 0.33$ (hexane/AcOEt 1:1); $=-12.5\left(c\right.$ 1.34, $\left.\mathrm{CH}_{2} \mathrm{Cl}_{2}\right)$; IR (film) v 3198, 2962, 2922, 2864, 1474, 1447, 1262, 1047, 900, 770, 696 $\mathrm{cm}^{-1}$; ${ }^{1} \mathrm{H}-\mathrm{NMR}\left(400 \mathrm{MHz}, \mathrm{CDCl}_{3}\right) \delta$ 7.42-7.37 (3H, m, ArH), 7.25-7.22 (3H, m, ArH), 7.17 (1H, td, $J$ $=7.4,1.4 \mathrm{~Hz}, \operatorname{ArH}), 7.10(1 \mathrm{H}, \mathrm{td}, J=7.5,1.5 \mathrm{~Hz}$, ArH), 7.00 (1H, dd, $J=7.7,1.4 \mathrm{~Hz}, \mathrm{ArH}), 6.09$ $(1 \mathrm{H}, \mathrm{t}, J=4.5 \mathrm{~Hz}, \mathrm{C}=\mathrm{CH}), 5.67(1 \mathrm{H}, \mathrm{d}, J=1.6 \mathrm{~Hz}$, $\mathrm{C}=\mathrm{CH} H), 5.35(1 \mathrm{H}, \mathrm{d}, J=1.6 \mathrm{~Hz}, \mathrm{C}=H \mathrm{H}), 4.58$ $(1 \mathrm{H}, \mathrm{q}, J=7.1 \mathrm{~Hz}, \mathrm{CH}), 3.49(1 \mathrm{H}, \mathrm{d}, J=8.1 \mathrm{~Hz}$, $\mathrm{NH}), 2.89(1 \mathrm{H}, \mathrm{ddd}, J=17.1,5.7,4.1 \mathrm{~Hz}, \mathrm{CH} H)$, $2.67(1 \mathrm{H}, \mathrm{ddd}, J=17.2,7.1,4.8 \mathrm{~Hz}, \mathrm{CHH}), 1.23$ $\left[9 \mathrm{H}, \mathrm{s}, \mathrm{C}\left(\mathrm{CH}_{3}\right)_{3}\right] ;{ }^{13} \mathrm{C}-\mathrm{NMR}\left(101 \mathrm{MHz}, \mathrm{CDCl}_{3}\right) \delta$ 147.9, 139.7, 139.6, 135.6, 133.4 (C), 128.3, 128.0, $127.7,127.3,126.7,126.6,126.3,125.1(\mathrm{CH})$, $115.4\left(\mathrm{CH}_{2}\right), 56.1(\mathrm{C}), 54.3(\mathrm{CH}), 32.6\left(\mathrm{CH}_{2}\right), 22.6$ $\left(\mathrm{CH}_{3}\right)$; LRMS (EI) m/z $295\left(\mathrm{M}^{+}-\mathrm{C}_{4} \mathrm{H}_{8}, 3 \%\right), 232$ (25), 231 (92), 230 (65), 229 (29), 228 (10), 215 (15), 153 (39), 152 (13), 104 (10), 103 (100), 77 (13), 71 (17), 69 (14), 57 (45), 55 (15), 43 (27), 41 (18).

$\left(1 \mathrm{~S}, \mathrm{R}_{S}\right)-\mathrm{N}-($ tert-Butanesulfinyl)-7-methoxy-4(1-phenylvinyl)-1,2-dihydronaphthalen-1-amine (12d): colourless oil; $R_{\mathrm{F}} 0.38$ (hexane/AcOEt 1:1); $=-13.5\left(c\right.$ 1.34, $\left.\mathrm{CH}_{2} \mathrm{Cl}_{2}\right)$; IR (film) v 3198, 3030, 2951, 2922, 2863, 2834, 1607, 1569, 1492, 1261, 1250, 1039, 897, 735, $696 \mathrm{~cm}^{-1} ;{ }^{1} \mathrm{H}-\mathrm{NMR}(400$ $\left.\mathrm{MHz}, \mathrm{CDCl}_{3}\right) \delta$ 7.42-7.37 (2H, m, ArH), 7.25-7.20 $(3 \mathrm{H}, \mathrm{m}, \mathrm{ArH}), 6.97(1 \mathrm{H}, \mathrm{dd}, J=2.7,0.8 \mathrm{~Hz}, \mathrm{ArH})$, $6.91(1 \mathrm{H}, \mathrm{d}, J=8.5 \mathrm{~Hz}, \mathrm{ArH}), 6.61(1 \mathrm{H}, \mathrm{dd}, J=$
8.5, 2.7 Hz, ArH), $5.97(1 \mathrm{H}, \mathrm{t}, J=4.5 \mathrm{~Hz}, \mathrm{C}=\mathrm{CH})$, $5.64(1 \mathrm{H}, \mathrm{d}, J=1.7 \mathrm{~Hz}, \mathrm{C}=\mathrm{CH} H), 5.34(1 \mathrm{H}, \mathrm{d}, J$ $=1.7 \mathrm{~Hz}, \mathrm{C}=\mathrm{CHH}), 4.54(1 \mathrm{H}, \mathrm{td}, J=8.2,5.7 \mathrm{~Hz}$, $\mathrm{CH}), 3.76\left(3 \mathrm{H}, \mathrm{s}, \mathrm{CH}_{3}\right), 3.48(1 \mathrm{H}, \mathrm{d}, J=8.5 \mathrm{~Hz}$, $\mathrm{NH}), 2.87(1 \mathrm{H}, \mathrm{ddd}, J=16.9,5.8,4.5 \mathrm{~Hz}, \mathrm{CH} H)$, $2.62(1 \mathrm{H}, \mathrm{ddd}, J=16.9,8.0,4.6 \mathrm{~Hz}, \mathrm{CHH}), 1.25$ $\left[9 \mathrm{H}, \mathrm{s}, \mathrm{C}\left(\mathrm{CH}_{3}\right)_{3}\right] ;{ }^{13} \mathrm{C}-\mathrm{NMR}\left(101 \mathrm{MHz}, \mathrm{CDCl}_{3}\right) \delta$ 158.7, 148.0, 139.8, 139.2, 137.7 (C), 128.3, 127.7, $126.6(\mathrm{CH}), 126.5(\mathrm{C}), 122.7(\mathrm{CH}), 115.2\left(\mathrm{CH}_{2}\right)$, 112.7, $112.3(\mathrm{CH}), 56.1(\mathrm{C}), 55.2(\mathrm{CH}), 54.9\left(\mathrm{CH}_{3}\right)$, $32.7\left(\mathrm{CH}_{2}\right), 22.7\left(\mathrm{CH}_{3}\right)$; LRMS (EI) $\mathrm{m} / z 277\left(\mathrm{M}^{+}\right.$ $\left.-\mathrm{C}_{4} \mathrm{H}_{8} \mathrm{OS}, 4 \%\right), 262$ (45), 261 (87), 260 (100), 229 (15), 215 (12), 202 (14), 183 (27), 103 (88), 97 (18), 85 (18), 83 (16), 77 (12), 71 (24), 70 (19), 69 (19), 57 (54), 55 (22), 43 (78).

(1S, $\left.\mathrm{R}_{S}\right)$-N-(tert-Butanesulfinyl)-4-[1-(4methoxyphenyl)vinyl]-1,2-dihydronaphthalen-1amine (12e): colourless oil; $R_{\mathrm{F}} 0.30$ (hexane/AcOEt $1: 1$ ); $=-13.1$ ( c 1.53, $\mathrm{CH}_{2} \mathrm{Cl}_{2}$ ); IR (film) v 3213, 2957, 2927, 2859, 1604, 1509, 1458, 1247, 1176, $1031,897,835,755,737 \mathrm{~cm}^{-1}$; ${ }^{1} \mathrm{H}-\mathrm{NMR}(400 \mathrm{MHz}$, $\left.\mathrm{CDCl}_{3}\right) \delta$ 7.40-7.37 (1H, m, ArH), 7.33 (2H, d, $J=$ $8.9 \mathrm{~Hz}, \mathrm{ArH}), 7.17$ (1H, td, $J=7.4,1.5 \mathrm{~Hz}, \mathrm{ArH})$, $7.11(1 \mathrm{H}, \mathrm{td}, J=7.5,1.5 \mathrm{~Hz}, \operatorname{ArH}), 7.00$ (1H, dd, $J$ $=7.7,1.4 \mathrm{~Hz}, \mathrm{ArH}), 6.77(2 \mathrm{H}, \mathrm{d}, J=8.8 \mathrm{~Hz}, \mathrm{ArH})$, $6.08(1 \mathrm{H}, \mathrm{t}, J=4.5 \mathrm{~Hz}, \mathrm{C}=\mathrm{CH}), 5.57(1 \mathrm{H}, \mathrm{d}, J=1.7$ $\mathrm{Hz}, \mathrm{C}=\mathrm{CH} H), 5.24(1 \mathrm{H}, \mathrm{d}, J=1.7 \mathrm{~Hz}, \mathrm{C}=\mathrm{C} H \mathrm{H})$, $4.58(1 \mathrm{H}, \mathrm{q}, J=7.2 \mathrm{~Hz}, \mathrm{CH}), 3.77\left(3 \mathrm{H}, \mathrm{s}, \mathrm{CH}_{3}\right), 3.48$ $(1 \mathrm{H}, \mathrm{d}, J=8.1 \mathrm{~Hz}, \mathrm{NH}), 2.88(1 \mathrm{H}, \mathrm{ddd}, J=17.2$, $5.8,4.2 \mathrm{~Hz}, \mathrm{CH} H), 2.66$ (1H, ddd, $J=17.1,7.3,4.8$ $\mathrm{Hz}, \mathrm{CHH}), 1.23\left[9 \mathrm{H}, \mathrm{s}, \mathrm{C}\left(\mathrm{CH}_{3}\right)_{3}\right] ;{ }^{13} \mathrm{C}-\mathrm{NMR}(101$ $\left.\mathrm{MHz}, \mathrm{CDCl}_{3}\right) \delta 159.3,147.2,139.8,135.6,133.5$, 132.2 (C), 127.9, 127.8, 126.6, 126.3, 124.8, 113.6 $(\mathrm{CH}), 113.5\left(\mathrm{CH}_{2}\right), 56.0(\mathrm{C}), 55.2(\mathrm{CH}), 54.4\left(\mathrm{CH}_{3}\right)$, $32.6\left(\mathrm{CH}_{2}\right), 22.6\left(\mathrm{CH}_{3}\right)$; LRMS (EI) $\mathrm{m} / z 277\left(\mathrm{M}^{+}\right.$ $\left.-\mathrm{C}_{4} \mathrm{H}_{8} \mathrm{OS}, 8 \%\right), 263$ (15), 261 (56), 260 (47), 259 (26), 153 (16), 135 (14), 133 (100), 70 (13), 57 (35), 43 (57).

(1S, $\left.\mathrm{R}_{S}\right)-\mathrm{N}$-(tert-Butanesulfinyl)-4-vinyl-1,2dihydronaphthalen-1-amine (12f): colourless oil; $R_{\mathrm{F}} 0.33$ (hexane/AcOEt 1:1); = -6.9 (c 1.57, $\mathrm{CH}_{2} \mathrm{Cl}_{2}$ ); IR (film) v 3208, 2955, 2864, 1736, 1474 , 
1450, 1362, 1241, 1046, 911, $747 \mathrm{~cm}^{-1}$; ${ }^{1} \mathrm{H}-\mathrm{NMR}$ $\left(300 \mathrm{MHz}, \mathrm{CDCl}_{3}\right) \delta$ 7.45-7.36 (2H, m, ArH), 7.37$7.19(2 \mathrm{H}, \mathrm{m}, \mathrm{ArH}), 6.62(1 \mathrm{H}, \mathrm{dd}, J=17.3,10.9 \mathrm{~Hz}$, $\left.\mathrm{CH}=\mathrm{CH}_{2}\right), 6.12(1 \mathrm{H}, \mathrm{t}, J=4.8 \mathrm{~Hz}, \mathrm{C}=\mathrm{CH}), 5.55$ $(1 \mathrm{H}, \mathrm{dd}, J=17.3,1.8 \mathrm{~Hz}, \mathrm{CH}=\mathrm{CH} H), 5.24(1 \mathrm{H}, \mathrm{dd}$, $J=10.9,1.7 \mathrm{~Hz}, \mathrm{CH}=\mathrm{CHH}), 4.45(1 \mathrm{H}, \mathrm{dt}, J=8.9$, $6.4 \mathrm{~Hz}, \mathrm{CH}), 3.43(1 \mathrm{H}, \mathrm{d}, J=9.1 \mathrm{~Hz}, \mathrm{NH}), 2.77(1 \mathrm{H}$, $\mathrm{dt}, J=17.2,5.2 \mathrm{~Hz}, \mathrm{CH} H), 2.63(1 \mathrm{H}, \mathrm{dt}, J=17.2$, $6.2 \mathrm{~Hz}, \mathrm{CHH}), 1.19\left[9 \mathrm{H}, \mathrm{s}, \mathrm{C}\left(\mathrm{CH}_{3}\right)_{3}\right] ;{ }^{13} \mathrm{C}-\mathrm{NMR}$ $\left(75 \mathrm{MHz}, \mathrm{CDCl}_{3}\right) \delta 136.5,136.1(\mathrm{C}), 134.7(\mathrm{CH})$, $133.2(\mathrm{C}), 127.9,127.5,126.8,124.4,122.6(\mathrm{CH})$, $116.1\left(\mathrm{CH}_{2}\right), 56.1(\mathrm{C}), 54.7(\mathrm{CH}), 32.5\left(\mathrm{CH}_{2}\right), 22.6$ $\left(\mathrm{CH}_{3}\right)$; LRMS (EI) $\mathrm{m} / z 171\left(\mathrm{M}^{+}-\mathrm{C}_{4} \mathrm{H}_{8} \mathrm{OS}, 1 \%\right), 168$ (30), 155 (22), 154 (100), 153 (71), 152 (29), 141 (11), 115 (14).

$\left(6 \mathrm{~S}, \mathrm{R}_{S}\right)-\mathrm{N}$-(tert-Butanesulfinyl)-9-(oct-1-en2-yl)-6,7-dihydro-5Hbenzo[7]anulen-7-amine (16): colourless oil; $R_{\mathrm{F}} 0.54$ (hexane/AcOEt 1:1); $=-9.5\left(c\right.$ 1.76, $\left.\mathrm{CH}_{2} \mathrm{Cl}_{2}\right)$; IR (film) v 3208, 2954, 2926, 2858, 1449, 1362, 1055, 900, 752, $730 \mathrm{~cm}^{-}$ '; ${ }^{1} \mathrm{H}-\mathrm{NMR}\left(400 \mathrm{MHz}, \mathrm{CDCl}_{3}\right) \delta$ 7.28-7.21 $(1 \mathrm{H}$, m, ArH), 7.20-7.17 (3H, m, ArH), 6.16 (1H, t, $J=$ $7.3 \mathrm{~Hz}, \mathrm{C}=\mathrm{CH}), 5.04-4.98(1 \mathrm{H}, \mathrm{m}, \mathrm{C}=\mathrm{CH} H), 4.92$ $(1 \mathrm{H}, \mathrm{d}, J=2.2 \mathrm{~Hz}, \mathrm{C}=\mathrm{C} H \mathrm{H}), 4.15-4.05(1 \mathrm{H}, \mathrm{m}$, $\mathrm{CH}), 3.16(1 \mathrm{H}, \mathrm{d}, J=6.7 \mathrm{~Hz}, \mathrm{NH}), 2.89(1 \mathrm{H}, \mathrm{dd}, J$ $=13.2,6.2 \mathrm{~Hz}, \mathrm{CH} H), 2.60(1 \mathrm{H}, \mathrm{dd}, J=13.2,5.3$ $\mathrm{Hz}, \mathrm{CHH}), 2.29-2.19\left(3 \mathrm{H}, \mathrm{m}, \mathrm{CH}_{2}, \mathrm{CH} H\right), 1.95-$ $1.85(1 \mathrm{H}, \mathrm{m}, \mathrm{CH}), 1.43-1.34\left(2 \mathrm{H}, \mathrm{m}, \mathrm{CH}_{2}\right), 1.32-$ $1.24\left(6 \mathrm{H}, \mathrm{m}, 3 \times \mathrm{CH}_{2}\right), 1.23\left[9 \mathrm{H}, \mathrm{s}, \mathrm{C}\left(\mathrm{CH}_{3}\right)_{3}\right], 0.92-$ $0.81\left(3 \mathrm{H}, \mathrm{m}, \mathrm{CH}_{3}\right) ;{ }^{13} \mathrm{C}-\mathrm{NMR}\left(101 \mathrm{MHz}, \mathrm{CDCl}_{3}\right) \delta$ 148.9, 145.0, 139.1, 137.5 (C), 129.2, 129.1, 127.0, 126.2, $123.1(\mathrm{CH}), 114.6\left(\mathrm{CH}_{2}\right), 63.4(\mathrm{CH}), 55.6$ (C), 39.9, 34.5, 34.1, 31.7, 28.9, $28.1\left(\mathrm{CH}_{2}\right), 22.6$ $\left(\mathrm{CH}_{3}\right), 22.6\left(\mathrm{CH}_{2}\right), 14.1\left(\mathrm{CH}_{3}\right)$; LRMS (EI) $\mathrm{m} / z 269$ $\left(\mathrm{M}^{+}-\mathrm{C}_{4} \mathrm{H}_{8} \mathrm{OS}, 2 \%\right), 267$ (16), 252 (29), 251 (38), 182 (32), 181 (49), 179 (13), 168 (26), 167 (100), 166 (18), 165 (33), 156 (16), 155 (26), 154 (16), 153 (28), 152 (19), 141 (36), 128 (24), 115 (21).

\section{RESULTS AND DISCUSSION}

Homopropargylamine derivatives $\mathbf{6 a}$ and $\mathbf{6 b}$ were prepared first starting from ortho-bromostyrene $\mathbf{2 a}$. The reaction of $\mathbf{2} \mathbf{a}$ with $n$-BuLi in THF (an aromatic organolithium compound is formed through a bromine-lithium exchange), followed by reaction with $N, N$-dimethylformamide (DMF) and final hydrolysis with watergave ortho-vinylbenzaldehyde (3a) in $89 \%$ yield. Condensation of aldehyde $3 \mathbf{a}$ with $(R)$ - $N$-tert-butanesulfinamide (1) in the presence of $\mathrm{Ti}(\mathrm{OEt})_{4}$ led to $N$-tert-butanesulfinyl imine $4 \mathbf{a}$ in $75 \%$ yield. Further indium promoted propargylation of $\mathbf{4 a}$ with trimethylsilylpropargyl bromide (5) gave rise to the expected compound 6a with high diastereoselectivity (>98:2) and yield (70\%). Finally, treatment of $\mathbf{6 a}$ with potassium carbonate in a mixture of THF and methanol for 12 hours led to the formation of terminal alkyne $\mathbf{6} \mathbf{b}$ in $89 \%$ yield (Figure 2 ).

Unfortunately, all the attempts to perform a ring-closing metathesis in enynes $\mathbf{6 a}$ and $\mathbf{6 b}$, under the reaction conditions described by Bolm in the synthesis of cyclic sulfoximines with Grubbs second generation catalyst failed to produce the desired cyclic dienes 7 (Furger and Bolm 2009). Instead of that, starting compounds were recovered unaltered (Figure 3).

We decided to study the enyne ring-closing metathesis in other compounds different to $\mathbf{6 a}$ and $\mathbf{6 b}$. These compounds were prepared in two synthetic operations starting from orthobromobenzaldehyde $\left(\mathbf{3} \mathbf{b}, \mathrm{R}^{2}=\mathrm{H}\right)$ and 2-bromo-5methoxybenzaldehyde $\left(\mathbf{3 c}, \mathrm{R}^{2}=\mathrm{OMe}\right)$. First, the palladium catalyzed Sonogashira coupling reaction of aromatic aldehydes $\mathbf{3}$ with terminal alkynes $\mathbf{8}$ led to ortho-alkynyl aldehydes in high yields. After that, enynes 11 were obtained by aminoallylation of aldehyde 9 by successive reaction with $(R)$ $N$-tert-butanesulfinamide (1) in the presence of $\mathrm{Ti}(\mathrm{OEt})_{4}$ at room temperature for 1 hour, followed by addition of indium metal and allyl bromide (10), 

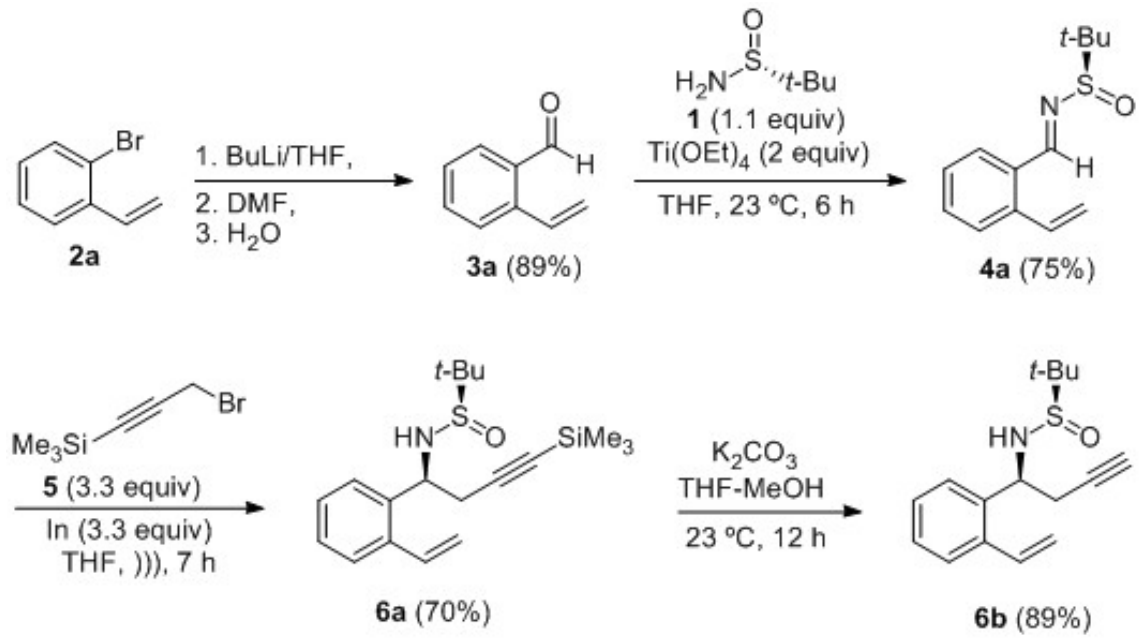

Figure 2 - Synthesis of enyne $\mathbf{6 b}$.

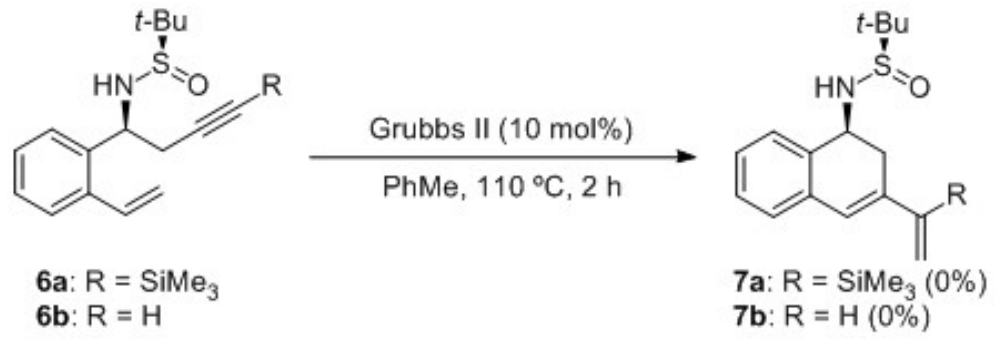

Figure 3 - Attempt to perform ring-closing metathesis of enynes 6.

and further reaction of the resulting mixture at 60 ${ }^{\circ} \mathrm{C}$ for 6 hours, in a one-pot process. The reaction was monitorized by TLC and it was not necessary to isolate the imine intermediate. The expected enynes $\mathbf{1 1}$ were obtained in good to moderate yields. The aminoallylation of aldehyde $\mathbf{9 d}$ with an electron-donating substituent at the aromatic ring took place in only $30 \%$ yield, leading to enyne 11d. On the other hand, enyne 11f was obtained in quantitative yield by desilylation of 11a (Table I).

The treatment of compounds $\mathbf{1 1}$ with a catalytic amount of Grubbs second generation catalyst in toluene a $110{ }^{\circ} \mathrm{C}$ for 1 hour led to the expected cyclic dienes $\mathbf{1 2}$ in variable yields, but for silylated derivative 11a $\left(\mathrm{R}^{1}=\mathrm{SiMe}_{3}\right)$ which did not undergo ring-closing metathesis and was recovered at the end of the reaction. The highest yields were obtained for enynes 11b $\left[\mathrm{R}^{1}=\left(\mathrm{CH}_{2}\right)_{5} \mathrm{Me}\right]$ and $\mathbf{1 1 f}$
$\left(\mathrm{R}^{1}=\mathrm{H}\right)$, which derived from aliphatic alkynes $\mathbf{8}$, leading to dienes $\mathbf{1 2 b}$ and $\mathbf{1 2 f}$, in 70 and 69\% yield, respectively. On the other hand, lower yields were found for enynes 11d and 11e with electrondonating methoxy groups in one of the aromatic rings (Table II).

Ring-closing metathesis of all enynes $\mathbf{1 1}$ shown on Table I produced six-membered cycles. With the aim of widen the scope of this reaction, we prepared an enyne system which after ring-closing metathesis would generate a seven-membered ring. For this purpose, aromatic aldehyde $\mathbf{9 b}$ was transformed into aldehyde 13. This homologation process was carried out through a Wittig reaction with methoxymethyl triphenylphosphonium chloride, followed by acidic hydrolysis. After that, the imine 14 was prepared in $64 \%$ yield by condensation with $(R)$ - $N$-tert-butanesulfinamide 
TABLE I

Synthesis of enynes 11 .

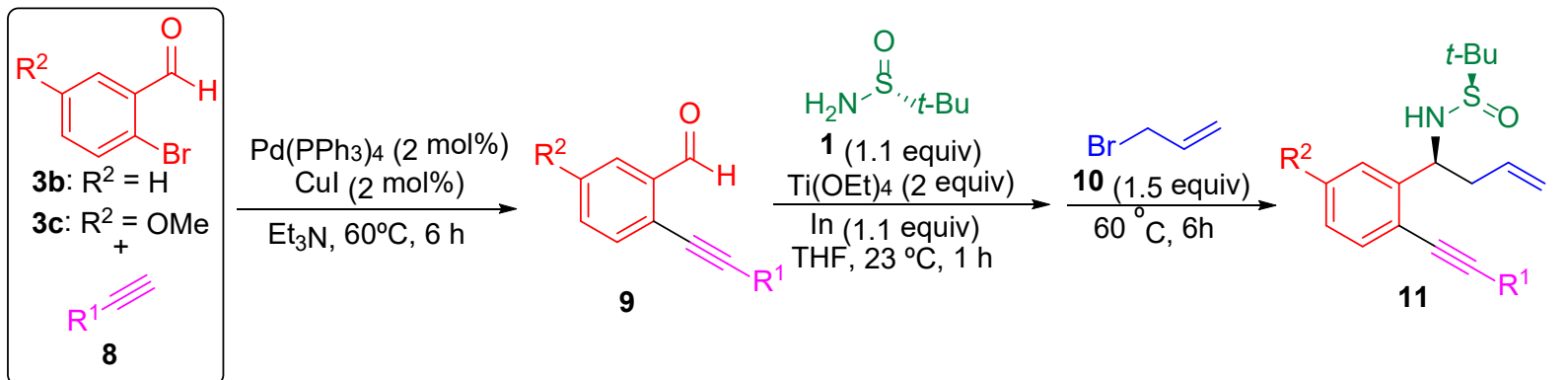

\begin{tabular}{|c|c|c|c|c|c|c|}
\hline \multirow{2}{*}{ Entry } & \multirow{2}{*}{$\mathrm{R}^{1}$} & \multirow{2}{*}{$\mathrm{R}^{2}$} & \multicolumn{2}{|c|}{ Aldehyde 9} & \multicolumn{2}{|c|}{ Enyne 11} \\
\hline & & & No. & Yield $(\%)^{\mathrm{a}}$ & No. & Yield $(\%)^{\mathrm{a}}$ \\
\hline 1 & $\mathrm{Me}_{3} \mathrm{Si}$ & $\mathrm{H}$ & $9^{a}$ & 50 & $11 \mathrm{a}$ & 55 \\
\hline 2 & $\mathrm{Me}\left(\mathrm{CH}_{2}\right)_{5}$ & $\mathrm{H}$ & $9 b$ & 88 & $11 b$ & 51 \\
\hline 3 & $\mathrm{Ph}$ & $\mathrm{H}$ & $9 \mathrm{c}$ & 98 & $11 \mathrm{c}$ & 82 \\
\hline 4 & $\mathrm{Ph}$ & $\mathrm{OMe}$ & 9d & 85 & 11d & 30 \\
\hline 5 & $4-\mathrm{MeOC}_{6} \mathrm{H}_{4}$ & $\mathrm{H}$ & $9 e$ & 93 & $11 \mathrm{e}$ & 64 \\
\hline 6 & $\mathrm{H}$ & $\mathrm{H}$ & & & $11 f$ & $>98^{\mathrm{b}}$ \\
\hline
\end{tabular}

a Isolated yield after column chromatography purification. ${ }^{\mathrm{b}}$ Compound $11 \mathrm{f}$ was obtained by desilylation of $11 \mathrm{a}$ under basis conditions.

TABLE II

Synthesis of dienes $12{ }^{\mathrm{a}}$<smiles>[R]C#Cc1ccc([R])cc1[C@H](CC=C)NS(=O)[R15]([R])([H])Br</smiles>

11<smiles>[R]C(=C)C1=CC[C@H](NS(=O)C([R14])(C)C)c2cc([R])ccc21</smiles>

12<smiles>C=CC1=CC[C@H](NS(=O)C(C)(C)C)c2ccccc21</smiles>

${ }^{a}$ Isolated yield after column chromatography purification. 
(1) in the presence of $\mathrm{Ti}(\mathrm{OEt})_{4}$. Allylation of this imine with allyl bromide (10) in the presence of indium metal gave the expected enyne $\mathbf{1 5}$ in 50\% yield. Finally, the ring-closing metathesis of $\mathbf{1 5}$ under the reactions conditions depicted on Table II led to diene $\mathbf{1 6}$ upon formation of a sevenmembered ring. Surprisingly this metathesis took place in higher yield (78\%) than for compounds $\mathbf{1 2}$, in spite of the higher stability of six-membered rings (Figure 4). Importantly, two stereogenic centers are present in dienes $\mathbf{1 2}$ and $\mathbf{1 6}$ (the carbon atom bonded to the nitrogen, and the sulfur atom), and are of potential interest in Diels-Alder reactions with different dienophiles, because high levels of regio- and stereoselectivity could be achieved in these transformations.

In conclusion, second generation Grubbs catalyst demonstrated to be effective in the ring- closing metathesis of $N$-tert-butanesulfinyl homoallylamine derivatives bearing ortho-alkynyl substituted aryl groups. The resulting dienes $\mathbf{1 2}$ and $\mathbf{1 6}$ are of potential interest in Diels-Alder reactions with different dienophiles, because high levels of regio- and stereoselectivity could be achieved in these transformations due to the presence of two stereogenic centers (the sulfur atom and the carbon atom bonded to the nitrogen). These chiral dienes are interesting precursors for the asymmetric synthesis of the skeleton present in some alkaloids, such as 17 (Foubelo and Yus 2014, 2016), and particularly in morphane derivatives (see, e.g. morphine; Figure 5) (Abate et al. 2012).

\section{ACKNOWLEDGMENTS}

We thank the continued financial support from our Ministerio de Ciencia e Innovación (MCINN;

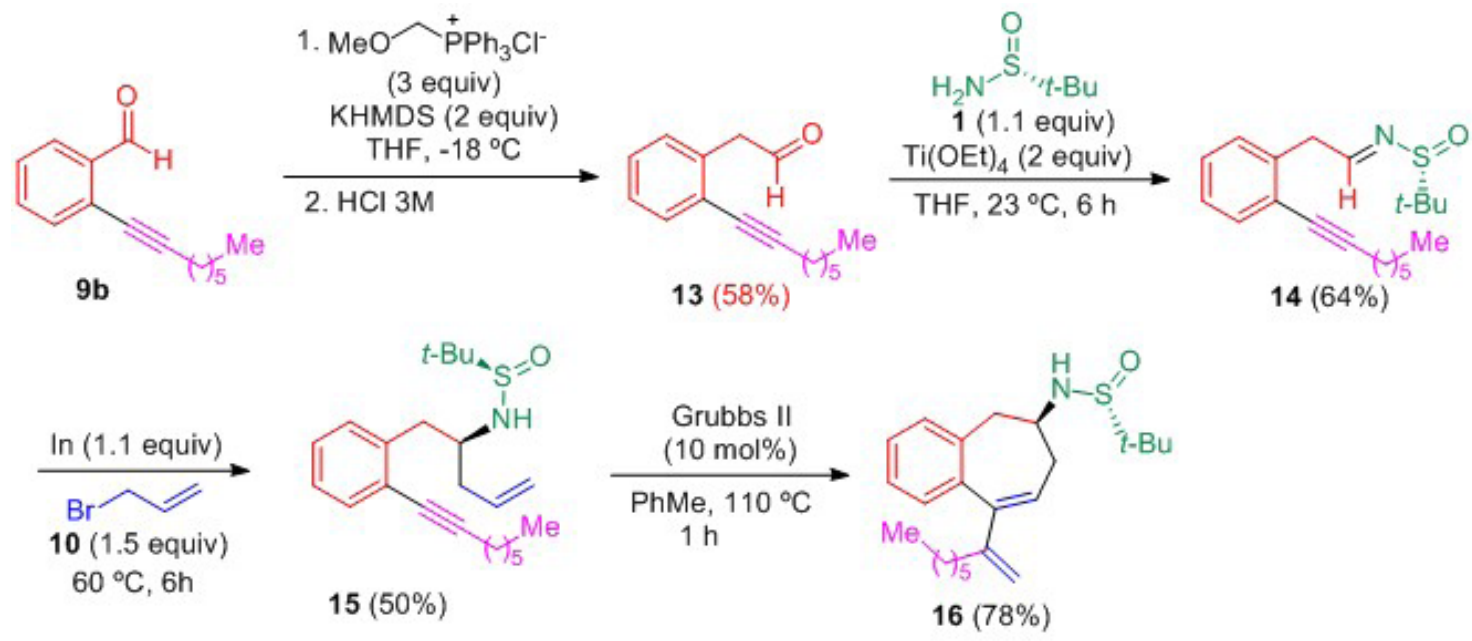

Figure 4 - Synthesis of diene 16.<smiles>[R]C(=C)C1=CC[C@H](NS(=O)C([R1])(C)C)c2cc([R])ccc21</smiles>

12

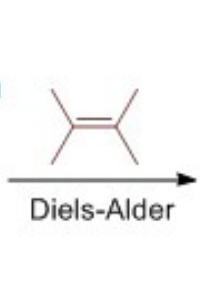

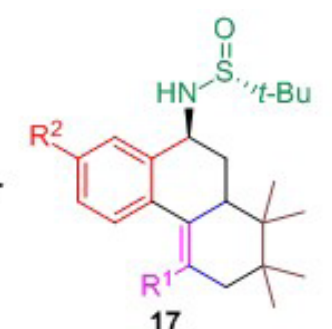

17

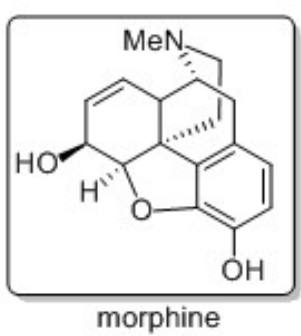

morphine

Figure 5 - Potential synthetic applications of dienes 12. 
CONSOLIDER INGENIO 2010-CDS2007-00006, CTQ2011-24165), the Ministerio de Economía y Competitividad (MINECO; projects CTQ201453695-P, CTQ2014-51912-REDC, CTQ201681797-REDC, CTQ2017-85093-P), FEDER, the Generalitat Valenciana (PROMETEO 2009/039, PROMETEOII/2014/017), and the University of

Alicante.

\section{REFERENCES}

ABATE C, PERRONE R AND BERNARDI F. 2012. Classes of sigma2 $(\sigma 2)$ receptor ligands: structure affinity relationship (SAfiR) studies and antiproliferative activity. Curr Pharm Design 18: 938-949.

BAUER RA, DIBLASI CM AND TAN DS. 2010. The tertbutylsulfinamide lynchpin in transition-metal-mediated multiscaffold library synthesis. Org Lett 12: 2084-2087.

FOUBELO F AND YUS M. 2004. Indium-mediated diastereoselective addition of allyl bromides to enantiomerically pure N-tert-butylsulfinyl aldimines. Tetrahedron: Asymmetry 15: 3823-3825.

FOUBELO F AND YUS M. 2014. Diastereoselective indiumpromoted allylation of chiral N-sulfinyl imines. Eur J Org Chem 485-491.

FOUBELO F AND YUS M. 2016. Synthesis of alkaloids by a diastereoselective allylation of chiral N-sulfinyl imines. Chem Today 34: 45-49.

FURGER B AND BOLM C. 2009. Ring-closing enyne metathesis (RCEYM) for the synthesis of cyclic sulfoximines. Synlett 1601-1604.

GARCIA-MUÑOZ MJ, FOUBELO F AND YUS M. 2016. Stereoselective synthesis of 2-(2-aminoalkyl)- and 1,3-disubstituted tetrahydro-1H-pyrido[4,3-b]- benzofuran and indole derivatives. J Org Chem 81: 10214-10226.
GARCIA-MUÑOZ MJ, ZACCONI F, FOUBELO F AND YUS M. 2013. Indium-promoted diastereo- and regioselective propargylation of chiral sulfinylimines. Eur J Org Chem 1287-1295.

HOVEYDA AH, GILLINGHAM DG, VAN VELDHUIZEN JJ, KATAOKA O, GARBER SB, KINGSBURY JS AND HARRITY JPA. 2004. Ru complexes bearing bidentate carbenes: from innocent curiosity to uniquely effective catalysts for olefin metathesis. Org Biomol Chem 2: 8-23.

KALIAPPAN KP. 2005. Recent advances in cascade enyne/ RCM in organic synthesis. Lett Org Chem 2: 678-686.

KATZ TJ AND SIVAVEC TM. 1985. Metal-catalyzed rearrangement of alkene-alkynes and the stereochemistry of metallacyclobutene ring opening. J Am Chem Soc 107: 737-738.

LAZARO R, BARRIO P, FINAMORE C, ROMAN R AND FUSTERO S. 2017. Homoallylic $o$-halobenzylamines: asymmetric diversity-oriented synthesis of benzo-fused cyclic amines. Struct Chem 28: 445-452.

RODRIGUEZ E, GRAYSON MN, ASENSIO A, BARRIO P, HOUK KN AND FUSTERO. 2016. Chiral Brønsted acidcatalyzed asymmetric allyl(propargyl)boration reaction of ortho-alkynyl benzaldehydes: synthetic applications and factors governing the enantioselectivity. ACS Catal 6: 2506-2514.

SCHMALZ HG. 1995. Catalytic ring-closing metathesis : A new, powerful technique for carbon-carbon coupling in organic synthesis. Angew Chem Int Ed 34: 1833-1836.

SIRVENT JA, FOUBELO F AND YUS M. 2012. Diastereoselective indium-mediated allylation of N-tertbutanesulfinyl ketimines: easy access to asymmetric quaternary stereocenters bearing nitrogen atoms. Chem Commun 48: 2543-2545.

VOUGIOUKALAKIS GC AND GRUBBS RH. 2010. Ruthenium-based heterocyclic carbene-coordinated olefin metathesis catalysts. Chem Rev 110: 1746-1787. 